\title{
Fam-Ti Catalyzed Enantioselective Alkynylation of Aldehydes
}

\author{
Hasan Koyuncu, Özdemir Dogan* \\ Department of Chemistry, Middle East Technical University, 06531 \\ Ankara, Turkey
}

General Data: All reactions were performed under a nitrogen or argon atmosphere in oven-dried glassware unless reported. Tetrahydrofuran (THF) was dried and distilled over Na-benzophenone prior to use. All reagents were purchased commercially and used without further purification unless stated otherwise. Chiral Ligand Fam (ferrocenyl substituted aziridinyl methanol) was weighed into a pre-dried flask attached to a vacuum line and heated with a heat gun to remove any moisture and then dissolved in dry THF before transferring into the reaction flask. Liquid aldehydes were used directly from their commercial bottles unless stated otherwise (only benzaldehyde and furfural were distilled before use). Solid aldehydes were also directly used from their commercial bottle (only 4chlorobenzaldehyde was distilled via bulb to bulb distillation). Solid aldehydes were weighed into a pre-dried sample tube which was attached to a vacuum-argon line. Sample tube was filled with argon atmosphere and aldehyde was dissolved in THF and transferred via syringe into the reaction medium. All of the products were purified by flash column chromatography on silica gel 60 (Merck, 230-400 mesh ASTM). TLC analyses were performed on $250 \mu \mathrm{m}$ Silica Gel 60 F254 plates and visualized by quenching of the UV fluorescence at $254 \mathrm{~nm}$. Unless indicated otherwise, ${ }^{1} \mathrm{H}-\mathrm{NMR}$ and ${ }^{13} \mathrm{C}-\mathrm{NMR}$ samples were prepared in 1:1 $\mathrm{CDCl}_{3}-\mathrm{CCl}_{4}$ and recorded at $400 \mathrm{MHz}$ and 100 $\mathrm{MHz}$, respectively. ${ }^{1} \mathrm{H}-\mathrm{NMR}$ data are reported as chemical shifts $(\delta, \mathrm{ppm})$ relative to tetramethylsilane $(\delta 0.00)$, multiplicity $(\mathrm{s}=$ singlet, $\mathrm{d}=$ doublet, $\mathrm{t}=$ triplet, $\mathrm{q}=$ quartet, $\mathrm{m}$ $=$ multiplet, $\mathrm{br}=$ broad singlet $)$, coupling constant $(\mathrm{Hz})$ and integration. Proton decoupled ${ }^{13} \mathrm{C}-\mathrm{NMR}$ data are reported as chemical shifts. Enantiomeric excess (ee) was determined by chiral HPLC analysis using a chiral stationary phase (Daicel Chiralcel OD-H, Daicel Chiralcel OJ-H or Daicel Chiralcel OD), eluting with $i$-PrOH-hexanes, and using UV detection at $254 \mathrm{~nm}$ unless stated otherwise.

\section{Representative general procedure for the addition of acetylene derivatives to aldehydes:}

Chiral ligand Fam-1 (0.0554 mmol, $20 \mathrm{mg}$ ) was weighed into a pre-dried flask which was attached to a vacuum-argon line. After vacuum, the flask was filled with argon and freshly distilled THF (1.15 mL, dried over sodium-benzophenone), Et 2 Zn (0.665 mmol, $1 \mathrm{M}$ solution in hexanes) and phenylacetylene $(0.776 \mathrm{mmol}, 85.2 \mu \mathrm{L})$ were added. This 
homogenous mixture was stirred at room temperature for 2 hours. Then, freshly distilled $\mathrm{Ti}\left(\mathrm{O}^{\mathrm{i}} \mathrm{Pr}\right)_{4}(0.139 \mathrm{mmol}, 41 \mu \mathrm{L})$ was added and this final mixture was stirred for 1 hour. At the end of this period, reaction mixture was cooled to $0{ }^{\circ} \mathrm{C}$ and aldehyde $(0.554 \mathrm{mmol})$ was added and the reaction was allowed to proceed for $5 \mathrm{~h}$ at this temperature. Saturated ammonium chloride $(3 \mathrm{~mL})$ was added to quench the reaction, and ethyl acetate $(3 \times 10$ $\mathrm{mL}$ ) was used for extraction. The organic phase was combined, dried over $\mathrm{Na}_{2} \mathrm{SO}_{4}$, and concentrated under reduced pressure. The residue was purified by flash column chromatography on silica gel with hexane/ethyl acetate (10:1) as the eluent to afford the propargylic alcohol. Enantiomeric excess valus were determined by chiral HPLC.<smiles>OC(=Cc1ccccc1)c1ccccc1</smiles>

1,3-Diphenyl-prop-2-yn-1-ol (5): 92\% isolated yield, 96\% ee determined by HPLC analysis (Chiralcel OD-H column 10\% IPA in hexanes, flow rate $=1.0 \mathrm{~mL} / \mathrm{min}, 254 \mathrm{~nm}$ ). Retention time: $\mathrm{t}_{\text {major }}=13.9 \mathrm{~min}$ and $\mathrm{t}_{\mathrm{minor}}=19.9 \mathrm{~min} .[\alpha]_{\mathrm{D}}{ }^{27}=+2.4\left(\mathrm{c}=1.67, \mathrm{CHCl}_{3}\right)$, lit ${ }^{1}$. $[\alpha]_{\mathrm{D}}{ }^{23}=+3.1\left(\mathrm{c}=3.1, \mathrm{CHCl}_{3}\right) .{ }^{1} \mathrm{H}$ NMR $\delta$ 7.59-7.57 (d, $\left.J=7.4 \mathrm{~Hz}, 2 \mathrm{H}, \mathrm{Ar}-\mathrm{H}\right), 7.46-7.43$ (m, 2H, Ar-H), 7.39-7.35 (m, 2H, Ar-H), 7.31-7.29 (m, 4H, Ar-H), 5.64 (s, 1H), 2.16 (bs, $1 \mathrm{H}) ;{ }^{13} \mathrm{C}$ NMR $\delta 140.8,131.8,128.6,128.5,128.3,128.2,126.7,122.6,89.0,86.6,65.0$.<smiles>COc1ccc(C(O)C#Cc2ccccc2)cc1</smiles>

1-(4-Methoxyphenyl)-3-phenyl-prop-2-yn-1-ol (6): 90\% isolated yield, 96\% ee determined by HPLC analysis (Chiralcel OD-H column 10\% IPA in hexanes, flow rate = $1.0 \mathrm{~mL} / \mathrm{min}, 254 \mathrm{~nm})$. Retention time: $t_{\text {major }}=17.5 \mathrm{~min}$ and $t_{\text {minor }}=28.9 \mathrm{~min}$. $[\alpha]_{\mathrm{D}}{ }^{28}=+5.6$ $\left(\mathrm{c}=2.26, \mathrm{CHCl}_{3}\right), \mathrm{lit}^{2} .[\alpha]_{\mathrm{D}}{ }^{15}=+3\left(\mathrm{c}=0.93, \mathrm{CHCl}_{3}\right) .{ }^{1} \mathrm{H} \mathrm{NMR} \delta 7.52-7.50(\mathrm{~d}, J=8.6 \mathrm{~Hz}$, 2H, Ar-H), 7.48-7.45 (m, 2H, Ar-H), 7.31-7.30 (m, 3H, Ar-H), 6.90-6.88 (d, $J=8.6 \mathrm{~Hz}$, 2H, Ar-H), 5.60 (d, $J=4.4 \mathrm{~Hz}, 1 \mathrm{H}), 3.81$ (s, 3H), 2.24 (d, $J=4.7 \mathrm{~Hz}, 1 \mathrm{H}) ;{ }^{13} \mathrm{C}$ NMR $\delta$ 159.7, 133.1, 131.7, 128.4, 128.2, 128.1, 122.7, 113.9, 89.2, 86.4, 64.6, 55.1.

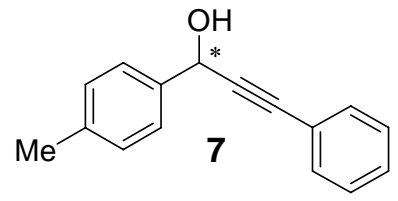

3-Phenyl-1-p-tolyl-prop-2-yn-ol (7): 93\% isolated yield, 92\% ee determined by HPLC analysis (Chiralcel OD-H column 10\% IPA in hexanes, flow rate $=1.0 \mathrm{~mL} / \mathrm{min}, 254 \mathrm{~nm}$ ). Retention time: $t_{\text {major }}=11.1 \mathrm{~min}$ and $t_{\text {minor }}=19.9 \mathrm{~min} .[\alpha]_{\mathrm{D}}{ }^{28}=+4.8\left(\mathrm{c}=1.00, \mathrm{CHCl}_{3}\right), \mathrm{lit}^{3}$. $[\alpha]_{\mathrm{D}}{ }^{20}=+4.02\left(\mathrm{c}=0.6, \mathrm{CHCl}_{3}\right) .{ }^{1} \mathrm{H}$ NMR $\delta$ 7.48-7.43 (m, 4H, Ph), 7.30-7.29 (m, 3H, $\mathrm{Ph}$ ), 7.19-7.17 (d, $J=7.9 \mathrm{~Hz}, 2 \mathrm{H}$ ), 5.61 (d, $J=6.1 \mathrm{~Hz}, 1 \mathrm{H}), 2.37$ (s, 3H), 2.12 (d, $J=6.3$ $\mathrm{Hz}, 1 \mathrm{H}) ;{ }^{13} \mathrm{C}$ NMR $\delta 137.9,131.8,129.3,128.4,128.2,126.7,122.6,89.1,86.5,64.9$.<smiles>OC(=Cc1ccccc1)c1ccc(Cl)cc1</smiles> 
1-(4-Chlorophenyl)-3-phenyl-prop-2-yn-1-ol (8): $91 \%$ isolated yield, $94 \%$ ee determined by HPLC analysis (Chiralcel OD-H column 10\% IPA in hexanes, flow rate $=$ $1.0 \mathrm{~mL} / \mathrm{min}, 254 \mathrm{~nm}$ ). Retention time: $\mathrm{t}_{\text {major }}=14.8 \mathrm{~min}$ and $\mathrm{t}_{\mathrm{minor}}=47.8 \mathrm{~min} .[\alpha]_{\mathrm{D}}{ }^{28}=+7.2$ (c= 1.25, $\left.\mathrm{CHCl}_{3}\right), \mathrm{lit}^{2} .[\alpha]_{\mathrm{D}}{ }^{15}=+6\left(\mathrm{c}=0.72, \mathrm{CHCl}_{3}\right) .{ }^{1} \mathrm{H}$ NMR $\delta 7.51(\mathrm{~d}, J=8.3 \mathrm{~Hz}, 2 \mathrm{H}$, Ar-H), 7.44-7.42 (m, 2H, Ar-H), 7.35-7.29 (m, 5H, Ar-H), 5.62 (s, 1H), 2.27 (bs, 1H);

${ }^{13}$ C NMR $\delta$ 139.2, 134.3, 131.7, 128.7, 128.6, 128.3, 128.0, 122.3, 88.4, 86.9, 64.3.<smiles>OC(C#Cc1ccccc1)c1ccc(Br)cc1</smiles>

1-(4-Bromophenyl)-3-phenyl-prop-2-yn-1-ol (9): $91 \%$ isolated yield, $92 \%$ ee determined by HPLC analysis (Chiralcel OD-H column 10\% IPA in hexanes, flow rate $=$ $1.0 \mathrm{~mL} / \mathrm{min}, 254 \mathrm{~nm})$. Retention time: $\mathrm{t}_{\text {major }}=14.5 \mathrm{~min}$ and $\mathrm{t}_{\text {minor }}=47.5 \mathrm{~min} .[\alpha]_{\mathrm{D}}{ }^{28}=+$ $6.2\left(\mathrm{c}=1.39, \mathrm{CHCl}_{3}\right), \mathrm{lit}^{2} .[\alpha]_{\mathrm{D}}{ }^{15}=+4\left(\mathrm{c}=0.76, \mathrm{CHCl}_{3}\right) .{ }^{1} \mathrm{H}$ NMR $\delta$ 7.51-7.41 (m, 6H, Ar-H), 7.33-7.27 (m, 3H, Ar-H), 5.60 (s, 1H), 2.35 (br, $1 \mathrm{H}) ;{ }^{13} \mathrm{C}$ NMR $\delta$ 139.7, 131.7, 131.6, 128.7, 128.3, 128.2, 122.4, 122.2, 88.4, 87.0, 64.3 .

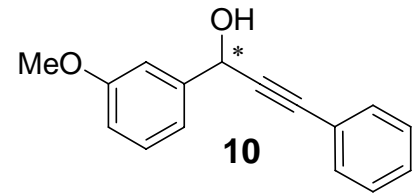

1-(3-Methoxyphenyl)-3-phenyl-prop-2-yn-1-ol (10): 91\% isolated yield, $96 \%$ ee determined by HPLC analysis (Chiralcel OD-H column 10\% IPA in hexanes, flow rate $=$ $1.0 \mathrm{~mL} / \mathrm{min}, 254 \mathrm{~nm})$. Retention time: $\mathrm{t}_{\text {major }}=24.8 \mathrm{~min}$ and $\mathrm{t}_{\mathrm{minor}}=38.3 \mathrm{~min} .[\alpha]_{\mathrm{D}}{ }^{28}=+$ $15.7\left(\mathrm{c}=1.03, \mathrm{CHCl}_{3}\right), \mathrm{lit}^{4} .[\alpha]_{\mathrm{D}}{ }^{27}=+11.4\left(\mathrm{c}=1.32, \mathrm{CHCl}_{3}\right) .{ }^{1} \mathrm{H}$ NMR $\delta$ 7.46-7.44 (m, 2H, Ar-H), 7.30-7.25 (m, 4H, Ar-H), 7.17-7.14 (m, 2H, Ar-H), 6.84 (d, J = 8.2 Hz, $1 \mathrm{H}$ ), 5.62 (d, $J=6.0 \mathrm{~Hz}, 1 \mathrm{H}), 3.83$ (s, 3H), $2.18(\mathrm{~d}, J=6.2 \mathrm{~Hz}, 1 \mathrm{H}) ;{ }^{13} \mathrm{C}$ NMR $\delta 159.9,142.3$, 131.8, 129.6, 128.5, 128.2, 122.6, 118.9, 114.1, 112.1, 88.9, 86.6, 64.9, 55.1.<smiles>OC(C#Cc1ccccc1)c1cccc(Br)c1</smiles>

1-(3-Bromophenyl)-3-phenyl-prop-2-yn-1-ol (11): 91\% isolated yield, 92\% ee determined by HPLC analysis (Chiralcel OD-H column $10 \%$ IPA in hexanes, flow rate $=$ $1.0 \mathrm{~mL} / \mathrm{min}, 254 \mathrm{~nm})$. Retention time: $\mathrm{t}_{\text {major }}=14.7 \mathrm{~min}$ and $\mathrm{t}_{\text {minor }}=53.8 \mathrm{~min} .[\alpha]_{\mathrm{D}}{ }^{25}=+$ $12.9\left(\mathrm{c}=1.52, \mathrm{CHCl}_{3}\right), \mathrm{lit}^{2} .[\alpha]_{\mathrm{D}}{ }^{15}=+6\left(\mathrm{c}=0.92, \mathrm{CHCl}_{3}\right) .{ }^{1} \mathrm{H}$ NMR $\delta 7.73(\mathrm{~s}, 1 \mathrm{H}, \mathrm{Ar}-\mathrm{H})$, 7.50 (d, $J=7.7 \mathrm{~Hz}, 1 \mathrm{H}, \mathrm{Ar}-\mathrm{H}), 7.45-7.43$ (m, 3H, Ar-H), 7.31-7.29 (m, 3H, Ar-H), 7.24 (t, $J=7.8 \mathrm{~Hz}, 1 \mathrm{H}, \mathrm{Ar}-\mathrm{H}), 5.61(\mathrm{~s}, 1 \mathrm{H}), 2.34$ (br, $1 \mathrm{H}) ;{ }^{13} \mathrm{C}$ NMR $\delta 142.9,131.8,131.3$, 130.0, 129.8, 128.7, 128.3, 125.2, 122.7, 122.2, 88.2, 87.1, 64.2.<smiles>COc1ccccc1C(=O)C#Cc1ccccc1</smiles>

1-(2-Methoxyphenyl)-3-phenyl-prop-2-yn-1-ol (12): 91\% isolated yield, 92\% ee determined by HPLC analysis (Chiralcel OD-H column 10\% IPA in hexanes, flow rate = 
$1.0 \mathrm{~mL} / \mathrm{min}, 254 \mathrm{~nm})$. Retention time: $\mathrm{t}_{\text {major }}=16.3 \mathrm{~min}$ and $\mathrm{t}_{\text {minor }}=19.9 \mathrm{~min} .[\alpha]_{\mathrm{D}}{ }^{28}=-$ $11.8\left(\mathrm{c}=1.22, \mathrm{CHCl}_{3}\right), \mathrm{lit}^{2} .[\alpha]_{\mathrm{D}}{ }^{18}=-8\left(\mathrm{c}=0.42, \mathrm{CHCl}_{3}\right) .{ }^{1} \mathrm{H}$ NMR $\delta 7.60(\mathrm{~d}, J=7.8 \mathrm{~Hz}$, 1H, Ar-H), 7.46-7.43 (m, 2H, Ar-H), 7.30-7.24 (m, 4H, Ar-H), 6.97 (t, $J=7.5 \mathrm{~Hz}, 1 \mathrm{H}$, Ar-H), 6.90 (d, $J=8.2 \mathrm{~Hz}, 1 \mathrm{H}, \mathrm{Ar}-\mathrm{H}), 5.86$ (br, $1 \mathrm{H}), 3.92$ (s, 3H), 2.85 (br, $1 \mathrm{H}) ;{ }^{13} \mathrm{C}$ NMR $\delta$ 156.8, 131.8, 129.5, 129.2, 128.2, 128.1, 128.0, 123.0, 121.0, 110.8, 88.7, 85.9, 61.5, 55.5 .<smiles>OC(C#Cc1ccccc1)c1ccc2ccccc2c1</smiles>

1-(Naphthalen-2-yl)-3-phenyl-prop-2-yn-1-ol (13): $90 \%$ isolated yield, $96 \%$ ee determined by HPLC analysis (Chiralcel OD-H column 10\% IPA in hexanes, flow rate $=$ $1.0 \mathrm{~mL} / \mathrm{min}, 254 \mathrm{~nm}$ ). Retention time: $\mathrm{t}_{\mathrm{major}}=16.5 \mathrm{~min}$ and $\mathrm{t}_{\mathrm{minor}}=49.7 \mathrm{~min} .[\alpha]_{\mathrm{D}}{ }^{28}=-7.8$ (c= 1.30, $\left.\mathrm{CHCl}_{3}\right), \mathrm{lit}^{2} .[\alpha]_{\mathrm{D}}{ }^{19}=-12\left(\mathrm{c}=2.42, \mathrm{CHCl}_{3}\right) .{ }^{1} \mathrm{H}$ NMR $\delta 8.00(\mathrm{~s}, 1 \mathrm{H}, \mathrm{Ar}-\mathrm{H})$, 7.84-7.78 (m, 3H, Ar-H), 7.68 (d, J = 8.5 Hz, 1H, Ar-H), 7.47-7.44 (m, 4H, Ar-H), 7.307.26 (m, 3H, Ar-H), 5.80 (s, $1 \mathrm{H}), 2.37$ (s, $1 \mathrm{H}) ;{ }^{13} \mathrm{C}$ NMR $\delta$ 138.1, 133.3, 133.2, 131.8, 128.5, 128.3, 127.7, 126.2, 125.5, 124.7, 122.6, 88.9, 86.9, 65.2.

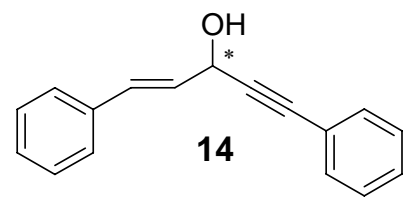

1,5-Diphenyl-pent-1-en-4-yn-3-ol (14): 87\% isolated yield 92\% ee determined by HPLC analysis (Chiralcel OD column 10\% IPA in hexanes, flow rate $=1.0 \mathrm{~mL} / \mathrm{min}, 254 \mathrm{~nm}$ ). Retention time: $\mathrm{t}_{\text {major }}=24.1 \mathrm{~min}$ and $\mathrm{t}_{\text {minor }}=66.3 \mathrm{~min} .[\alpha]_{\mathrm{D}}{ }^{26}=+0.5\left(\mathrm{c}=1.25, \mathrm{CHCl}_{3}\right)$, lit ${ }^{2} .[\alpha]_{\mathrm{D}}{ }^{15}=+1\left(\mathrm{c}=2.58, \mathrm{CHCl}_{3}\right) .{ }^{1} \mathrm{H}$ NMR $\delta$ 7.45-7.43 (m, 2H. Ar-H), $7.39(\mathrm{~d}, J=7.5$ $\mathrm{Hz}, 2 \mathrm{H}, \mathrm{Ar}-\mathrm{H}), 7.30-7.20$ (m, 6H, Ar-H), 6.79 (d, $J=15.8 \mathrm{~Hz}, 1 \mathrm{H}), 6.34$ (dd, $J=15.8$ \& $5.9 \mathrm{~Hz}, 1 \mathrm{H}), 5.23(\mathrm{~d}, J=5.5 \mathrm{~Hz}, 1 \mathrm{H}), 2.08(\mathrm{br}, 1 \mathrm{H}) ;{ }^{13} \mathrm{C}$ NMR $\delta 136.2,131.9,131.8$, 128.5, 128.4, 128.3, 128.2, 128.0, 126.8, 122.6, 88.2, 86.4, 63.4.

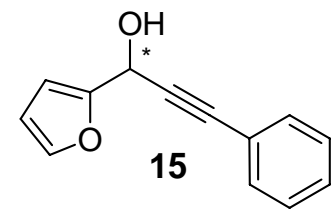

1-(Furan-2-yl)-3-phenyl-prop-2-yn-1-ol (15): 77\% isolated yield, 96\% ee determined by HPLC analysis (Chiralcel OD-H column 10\% IPA in hexanes, flow rate $=1.0$ $\mathrm{mL} / \mathrm{min}, 254 \mathrm{~nm})$. Retention time: $\mathrm{t}_{\text {major }}=13.4 \mathrm{~min}$ and $\mathrm{t}_{\text {minor }}=27.1 \mathrm{~min} .[\alpha]_{\mathrm{D}}{ }^{28}=+10.4$ (c= 1.12, $\left.\mathrm{CHCl}_{3}\right), \mathrm{lit}^{5} .[\alpha]_{\mathrm{D}}{ }^{25}=+34\left(\mathrm{c}=0.58, \mathrm{CHCl}_{3}\right) .{ }^{1} \mathrm{H}$ NMR $\delta$ 7.46-7.44 (m, 2H, ArH), 7.40 (s, $1 \mathrm{H}, \mathrm{Ar}-\mathrm{H}), 7.30-7.24$ (m, 3H, Ar-H), 6.48 (d, $J=2.8 \mathrm{~Hz}, 1 \mathrm{H}), 6.34(\mathrm{~s}, 1 \mathrm{H})$, $5.63(\mathrm{~s}, 1 \mathrm{H}), 2.46$ (br, $1 \mathrm{H}) ;{ }^{13} \mathrm{C}$ NMR $\delta$ 153.1, 142.8, 131.8, 128.6, 128.2, 122.3, 110.4, 107.7, 86.4, 85.7, 58.6.

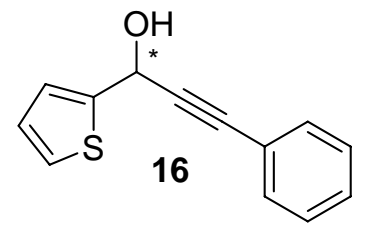


3-Phenyl-1-(thiophen-2-yl)prop-2-yn-1-ol (16): 73 \% isolated yield 96\% ee determined by HPLC analysis (Chiralcel OD-H column 10\% IPA in hexanes, flow rate $=1.0$ $\mathrm{mL} / \mathrm{min}, 254 \mathrm{~nm})$. Retention time: $\mathrm{t}_{\text {major }}=11.3 \mathrm{~min}$ and $\mathrm{t}_{\mathrm{minor}}=21.1 \mathrm{~min} .[\alpha]_{\mathrm{D}}{ }^{28}=+20.7$ (c= 1.07, $\left.\mathrm{CHCl}_{3}\right), \mathrm{lit}^{5} .[\alpha]_{\mathrm{D}}=+20\left(\mathrm{c}=0.53, \mathrm{CHCl}_{3}\right) .{ }^{1} \mathrm{H}$ NMR $\delta$ 7.41-7.39 (m, 2H, Ar-H), 7.25-7.21 (m, 4H, Ar-H), 7.15 (d, $J=3.2 \mathrm{~Hz}, 1 \mathrm{H}, \mathrm{Ar}-\mathrm{H}), 6.91$ (t, $J=4.2 \mathrm{~Hz}, 1 \mathrm{H}, \mathrm{Ar}-\mathrm{H})$, 5.77 (br s, 1H), 2.27 (br s, $1 \mathrm{H}$ ); ${ }^{13} \mathrm{C}$ NMR $\delta 144.9,131.8,128.7,128.3,126.7,126.0$, 125.5, 122.3, 88.2, 86.0, 60.7 .<smiles>OC(=Cc1ccccc1)C1CCCCC1</smiles>

1-Cyclohexyl-3-phenyl-prop-2-yn-1-ol (17): 96\% isolated yield 86\% ee determined by HPLC analysis (Chiralcel OD-H column $10 \%$ IPA in hexanes, flow rate $=1.0 \mathrm{~mL} / \mathrm{min}$, $254 \mathrm{~nm})$. Retention time: $\mathrm{t}_{\mathrm{major}}=7.7 \mathrm{~min}$ and $\mathrm{t}_{\mathrm{minor}}=14.9 \mathrm{~min} .[\alpha]_{\mathrm{D}}{ }^{27}=-8.8(\mathrm{c}=1.29$, $\left.\mathrm{CHCl}_{3}\right), \mathrm{lit}^{6} .[\alpha]_{\mathrm{D}}{ }^{26}=-9.2\left(\mathrm{c}=1.0, \mathrm{CHCl}_{3}\right) .{ }^{1} \mathrm{H}$ NMR $\delta$ 7.40-7.38 (m, 2H, Ar-H), 7.287.26 (m, 3H, Ar-H), 4.33 (d, $J=5.9$ Hz, $1 \mathrm{H}, \mathrm{Ar}-\mathrm{H}), 1.93-1.91(\mathrm{~m}, 2 \mathrm{H}), 1.81-1.78$ (m, $2 \mathrm{H}), 1.72-1.58(\mathrm{~m}, 2 \mathrm{H}), 1.34-1.08$ (m, 5H); ${ }^{13} \mathrm{C}$ NMR $\delta 131.7,128.2$, 122.9, 89.4, 85.7, 67.5, 44.3, 28.7, 28.3, 26.5, 26.0.

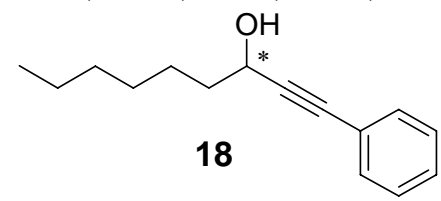

1-Phenylnon-1-yn-3-ol (18): 93\% isolated yield 88\% ee determined by HPLC analysis (Chiralcel OD-H column 10\% IPA in hexanes, flow rate $=1.0 \mathrm{~mL} / \mathrm{min}, 254 \mathrm{~nm}$ ). Retention time: $t_{\text {major }}=7.2 \mathrm{~min}$ and $\mathrm{t}_{\text {minor }}=16.5 \mathrm{~min}$. $[\alpha]_{\mathrm{D}}{ }^{26}=-4.6\left(\mathrm{c}=1.41, \mathrm{CHCl}_{3}\right)$, lit ${ }^{7}$. $[\alpha]_{\mathrm{D}}{ }^{23}=-1.5\left(\mathrm{c}=0.69, \mathrm{CHCl}_{3}\right) .{ }^{1} \mathrm{H}$ NMR $\delta$ 7.40-7.37 (m, 2H, Ar-H), 7.28-7.25 (m, 3H, Ar-H), 4.55 (t, $J=6.5 \mathrm{~Hz}, 1 \mathrm{H}), 1.85$ (bs, $1 \mathrm{H}), 1.82-1.71(\mathrm{~m}, 2 \mathrm{H}), 1.56-1.47$ (m, $2 \mathrm{H})$, 1.40-1.32 (m, 6H), 0.89 (t, $J=6.7 \mathrm{~Hz}, 3 \mathrm{H}) ;{ }^{13} \mathrm{C}$ NMR $\delta 131.7,128.2,122.8,90.4,84.8$, 62.9, 37.9, 31.8, 29.0, 25.2, 22.6, 14.1 .

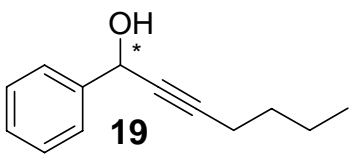

1-Phenylhept-2-yn-1-ol (19): 80\% isolated yield 94\% ee determined by HPLC analysis (Chiralcel OD-H column 6\% EtOH in hexanes, flow rate $=1.0 \mathrm{~mL} / \mathrm{min}, 214 \mathrm{~nm}$ ). Retention time: $\mathrm{t}_{\mathrm{minor}}=6.7 \mathrm{~min}$ and $\mathrm{t}_{\text {major }}=10.2 \mathrm{~min}$. $[\alpha]_{\mathrm{D}}{ }^{28}=+16.8\left(\mathrm{c}=1.13, \mathrm{CHCl}_{3}\right)$, lit ${ }^{8}$. $[\alpha]_{\mathrm{D}}=+5.44\left(\mathrm{c}=5.08, \mathrm{CHCl}_{3}\right) .{ }^{1} \mathrm{H}$ NMR $\delta 7.50(\mathrm{~d}, J=7.4 \mathrm{~Hz}, 2 \mathrm{H}, \mathrm{Ar}-\mathrm{H}), 7.35-7.24(\mathrm{~m}$, 3H, Ar-H), 5.39 (bs, 1H), 2.27 (dt, $J=1,8 \& 7.0 \mathrm{~Hz}, 2 \mathrm{H}$ ), 2.01 (bs, 1H), 1.49-1.39 (m, $2 \mathrm{H}), 1.57-1.50(\mathrm{~m}, 2 \mathrm{H}), 0.93$ (t, $J=7.2 \mathrm{~Hz}, 3 \mathrm{H}) ;{ }^{13} \mathrm{C}$ NMR $\delta 141.3,128.4,128.0,126.6$, 87.3, 80.3, 64.7, 30.7, 22.0, 18.5, 13.6 .

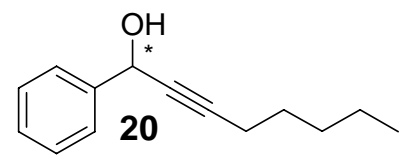

1-Phenyloct-2-yn-1-ol (20): 84\% isolated yield 94\% ee determined by HPLC analysis (Chiralcel OD-H column 6\% EtOH in hexanes, flow rate $=1.0 \mathrm{~mL} / \mathrm{min}, 214 \mathrm{~nm}$ ). Retention time: $t_{\text {minor }}=6.5$ min and $t_{\text {major }}=9.9$ min. $[\alpha]_{D}^{28}=+15.5\left(c=1.14, \mathrm{CHCl}_{3}\right)$, lit ${ }^{9}$. 
$[\alpha]_{\mathrm{D}}=+16.1(\mathrm{c}=1.1, \mathrm{EtOH}) .{ }^{1} \mathrm{H}$ NMR $\delta 7.50(\mathrm{~d}, J=7.3 \mathrm{~Hz}, 2 \mathrm{H}, \mathrm{Ar}-\mathrm{H}), 7.36-7.24(\mathrm{~m}$, 3H, Ar-H), 5.40 (bs, $1 \mathrm{H}), 2.25$ (dt, $J=1,8 \& 7.1 \mathrm{~Hz}, 2 \mathrm{H}$ ), 2.01 (bs, 1H), 1.59-1.49 (m, $2 \mathrm{H}), 1.42-1.29(\mathrm{~m}, 4 \mathrm{H}), 0.91(\mathrm{t}, J=7.0 \mathrm{~Hz}, 3 \mathrm{H}) ;{ }^{13} \mathrm{C}$ NMR $\delta 141.4,128.4,128.0,126.6$, 87.4, 80.3, 64.7, 31.1, 28.3, 22.2, 18.8, 14.0.

\section{References}

(1) Takita, R.; Yakura, K.; Ohshima, T.; Shibasaki, M. J. Am. Chem. Soc. 2005, 127, 13760.

(2) Xu, Z.; Chen, C.; Xu, J.; Miao, M.; Yan, W.; Wang, R. Org. Lett. 2004, 6, 1193.

(3) Fang, T.; Du, D. M.; Lu, S.F.; Xu, J. Org. Lett. 2005, 7, 2081.

(4) Pu, L.; Moore, D. Org. Lett. 2002, 4, 1855.

(5) Blay, G.; Fernandez, I.; Aleixandre, A. M.; Pedro, J. R. J. Org. Chem. 2006, 71, 6674.

(6) Frantz, D. E.; Fassler, R.; Carreira, E. M. J. Am. Chem. Soc. 2000, 122, 1806.

(7) Emmerson, D. P. G.; Hems, P. W.; Davis, B. G. Org. Lett. 2006, 8, 207.

(8) Niwa, S.; Soai, K. J. Chem. Soc., Perkin Trans. 1 1990, 937.

(9) Corey, E. J.; Cimprich, K. A. J. Am. Chem. Soc. 1994, 116, 3151. 

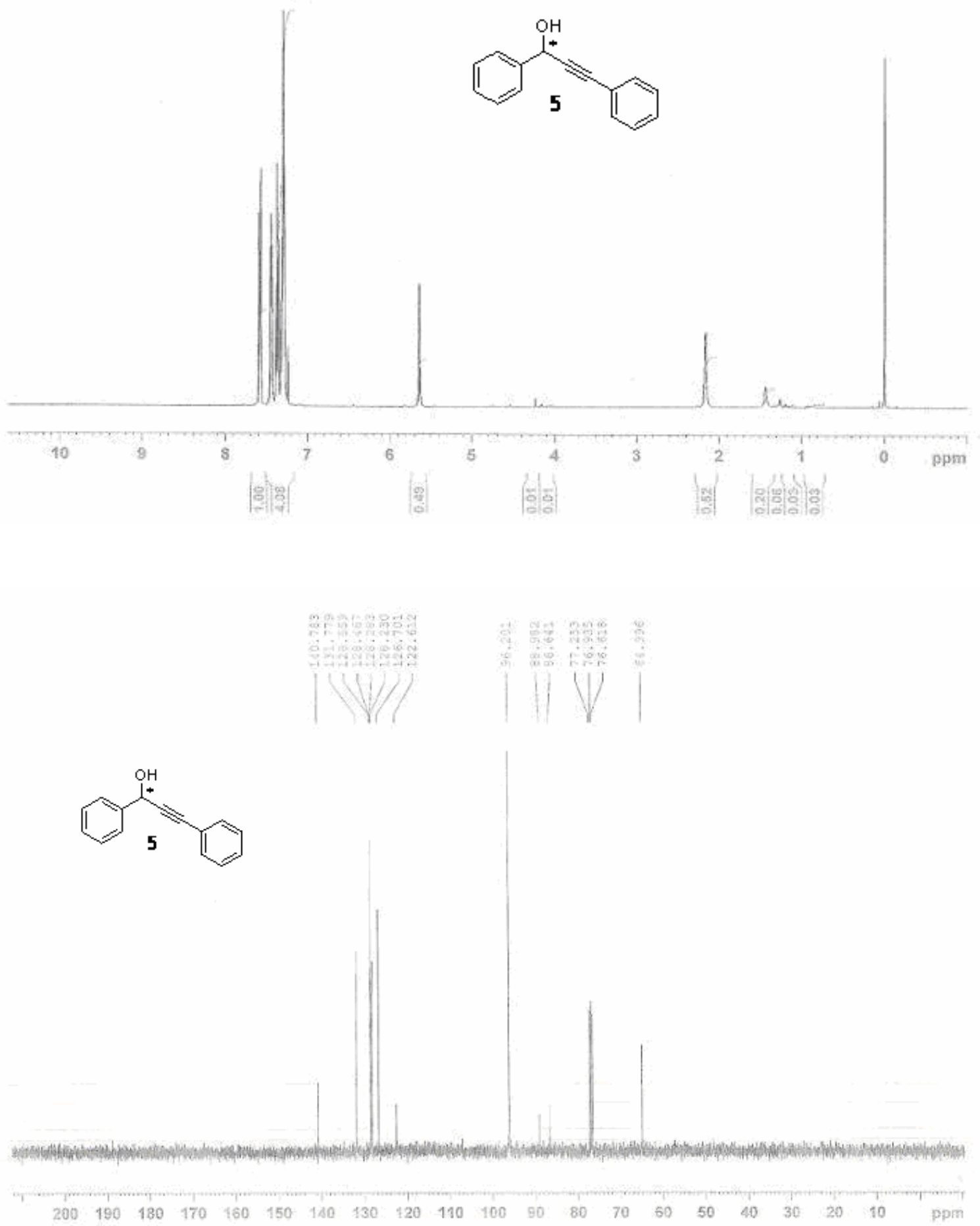

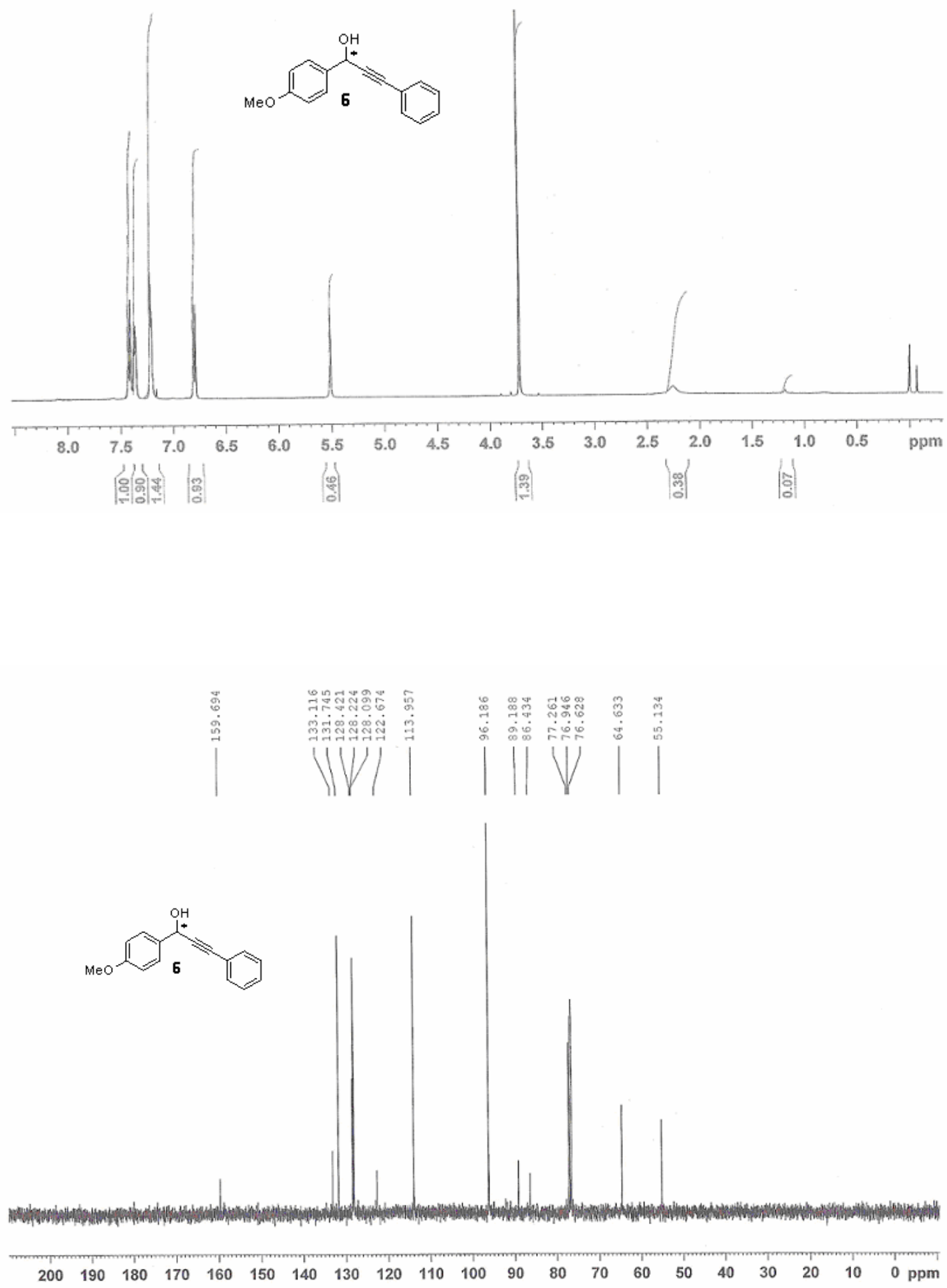


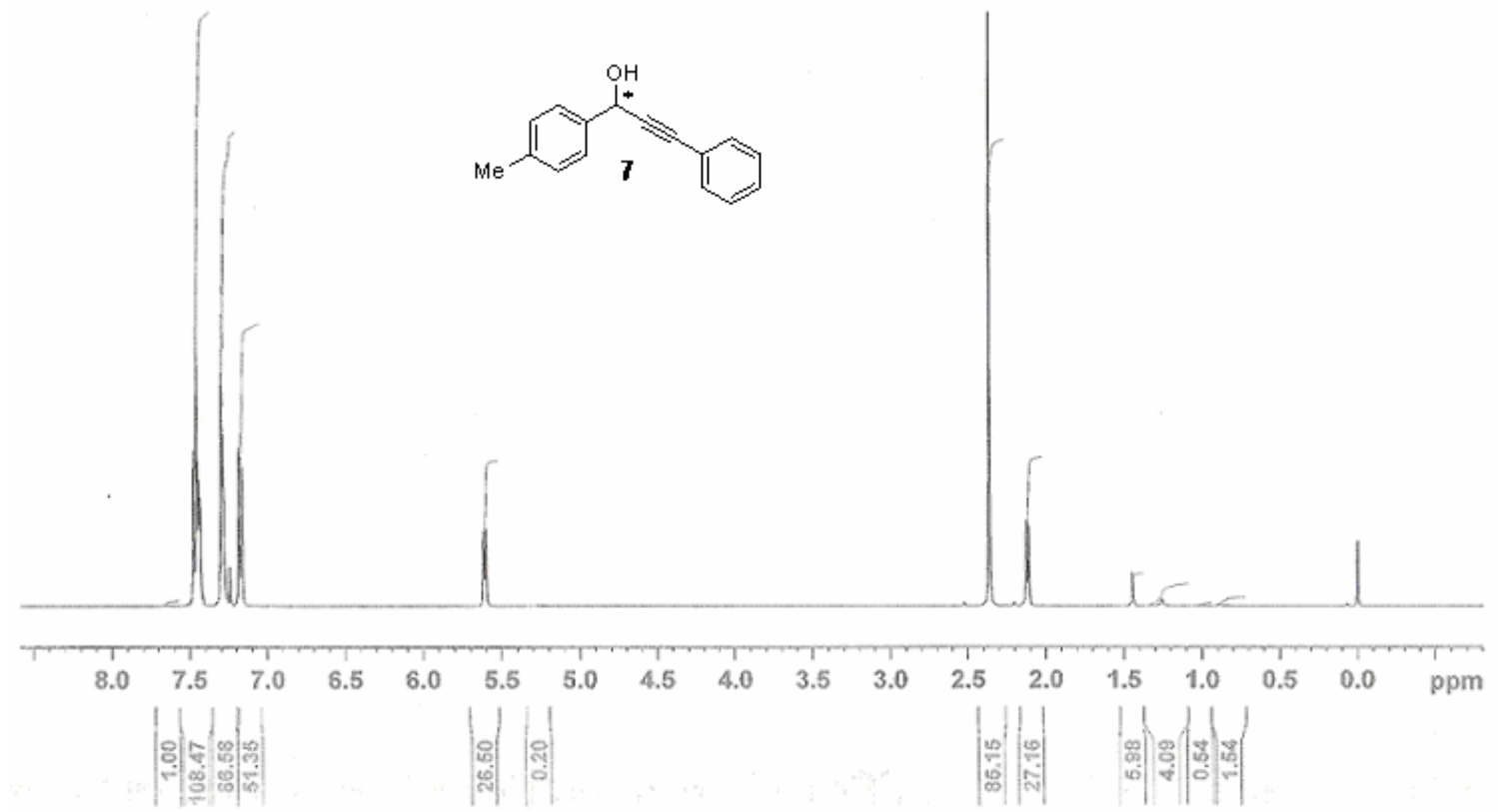

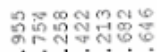

जि

IVWII

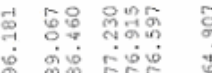
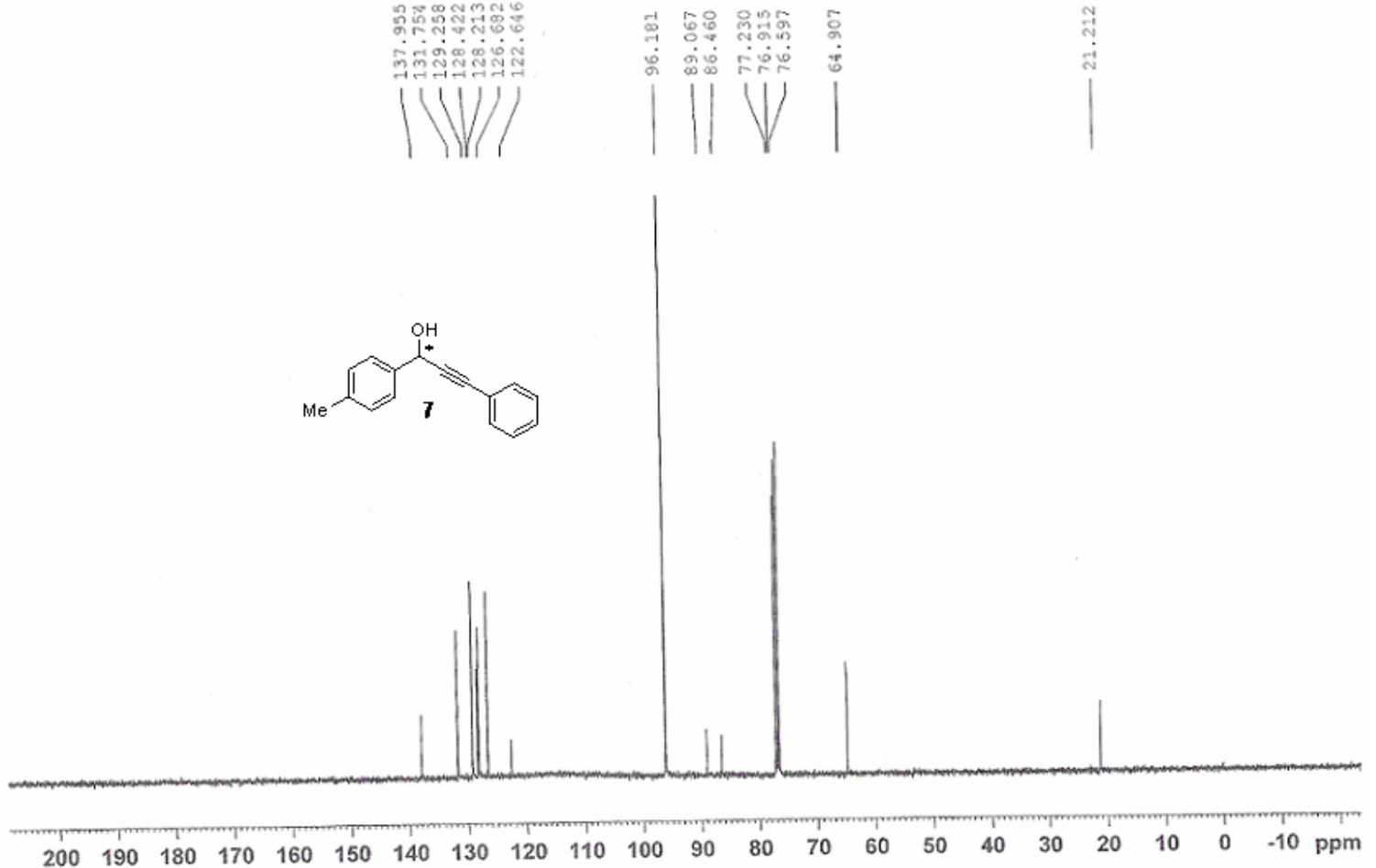

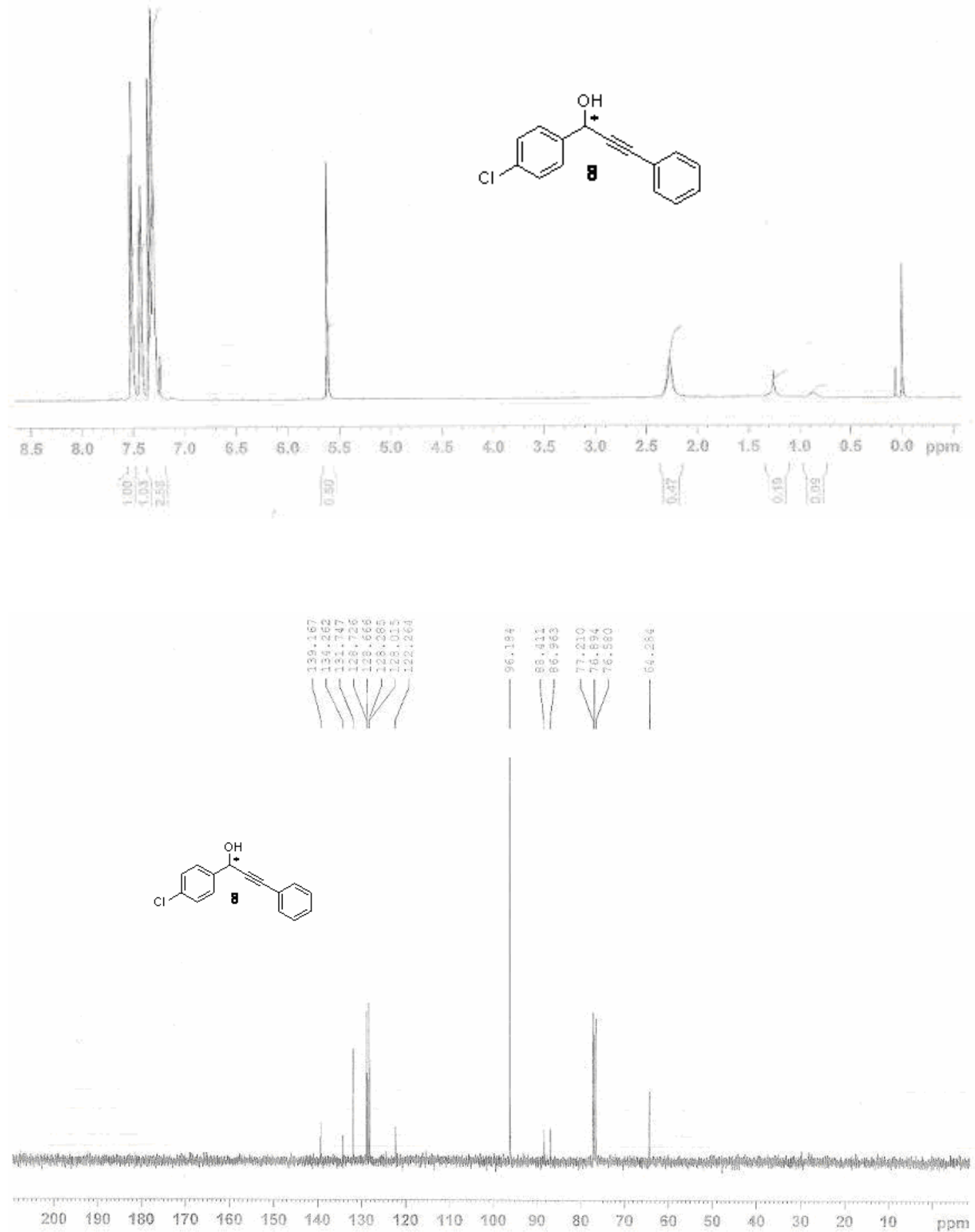

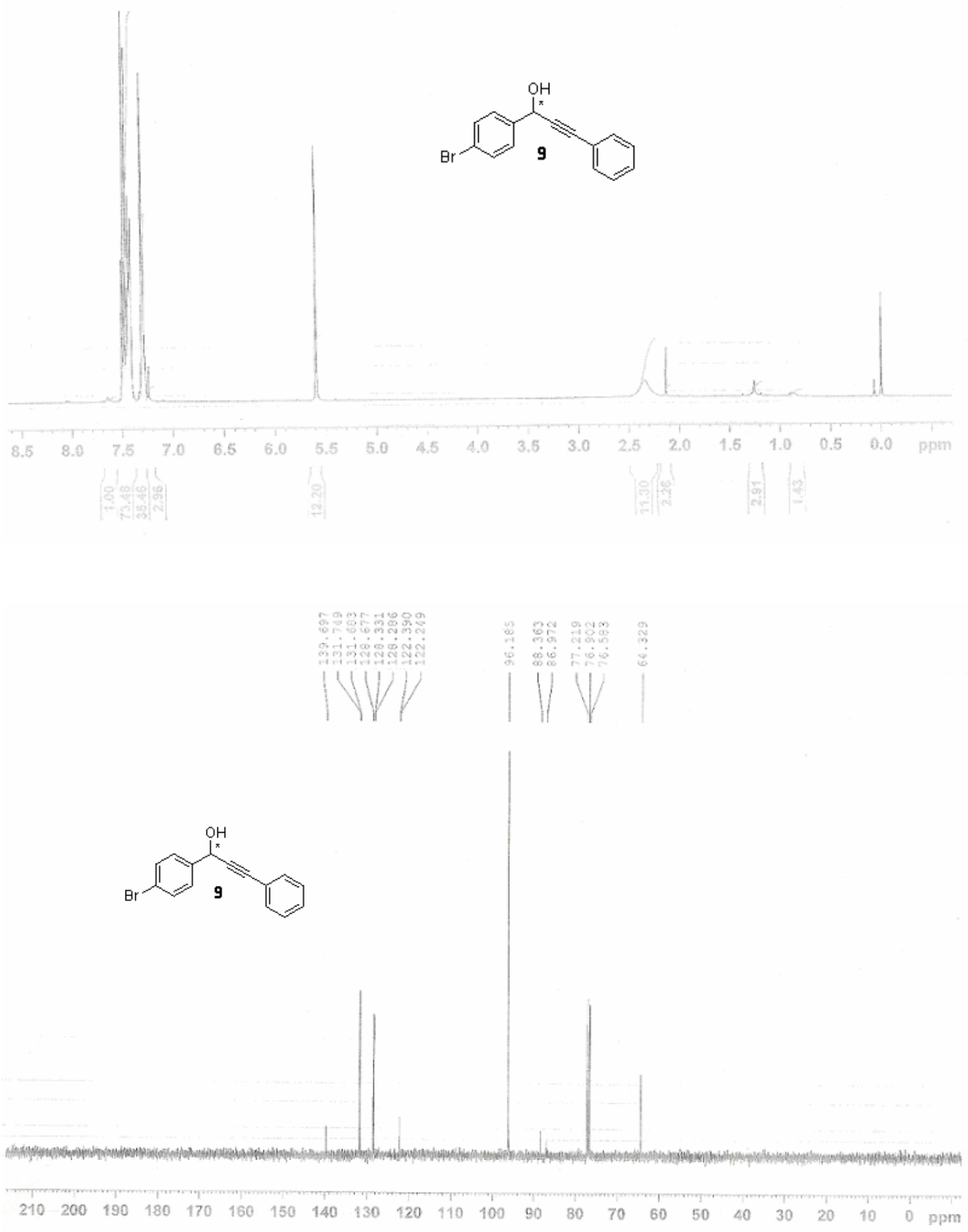

11 

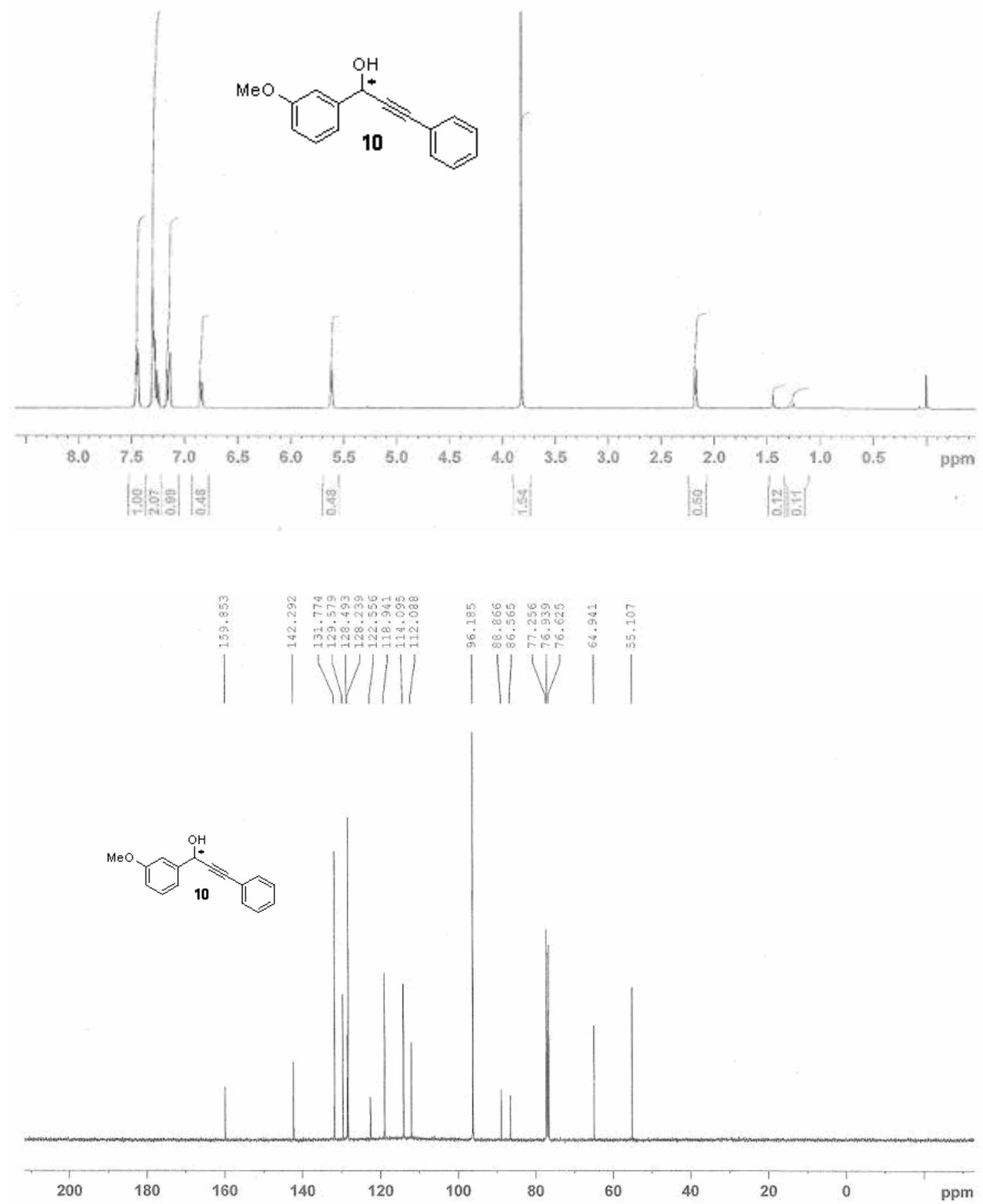

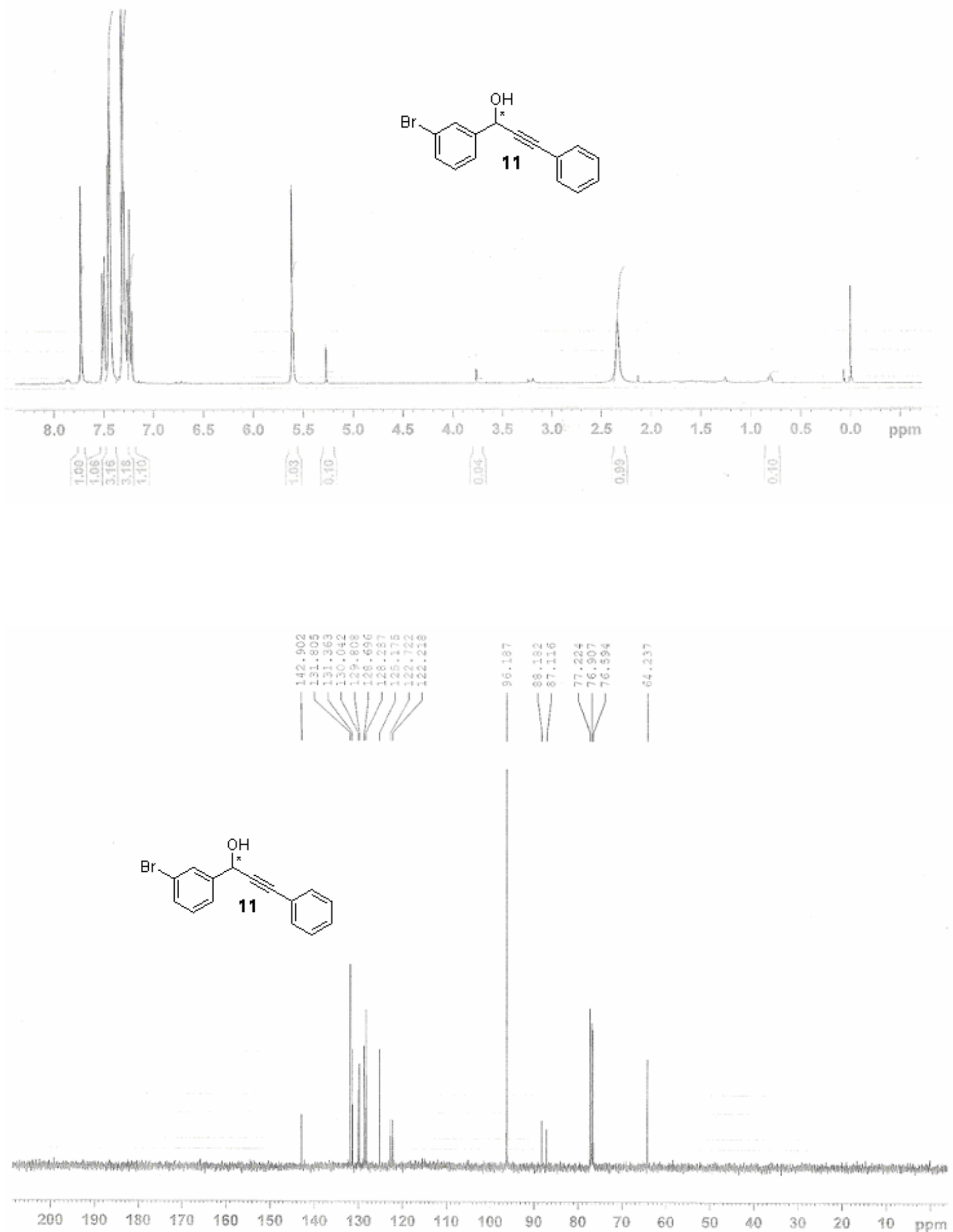

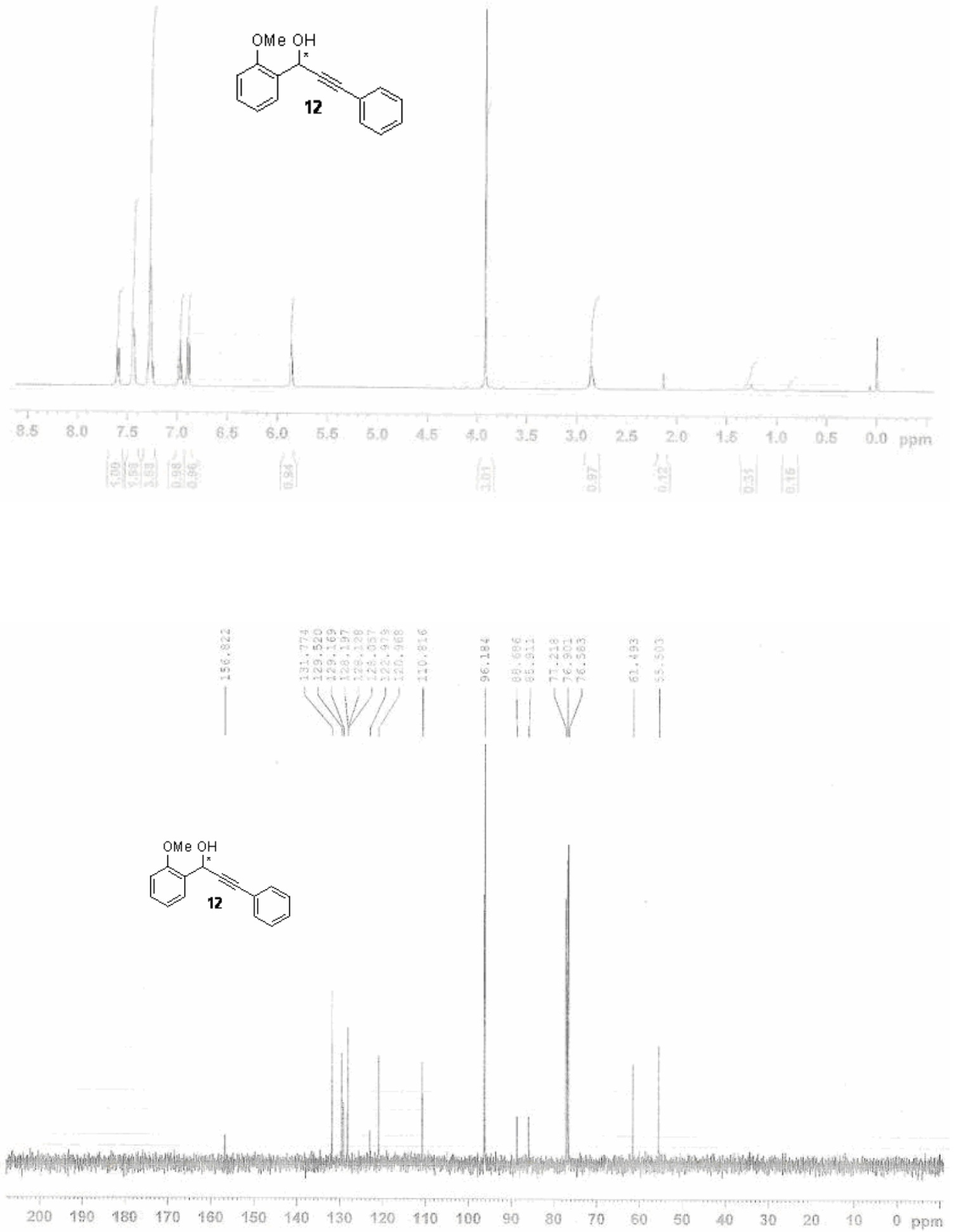

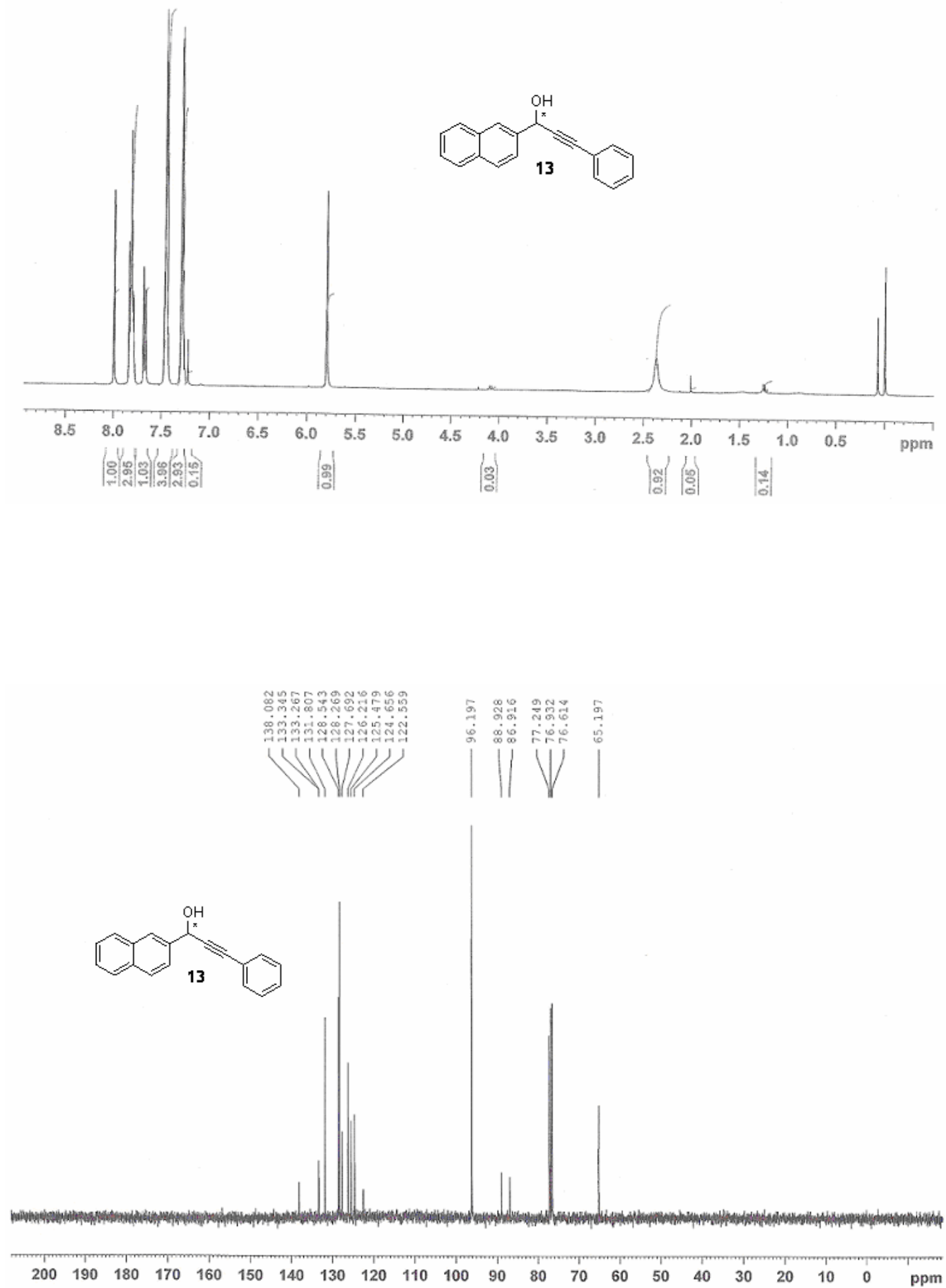

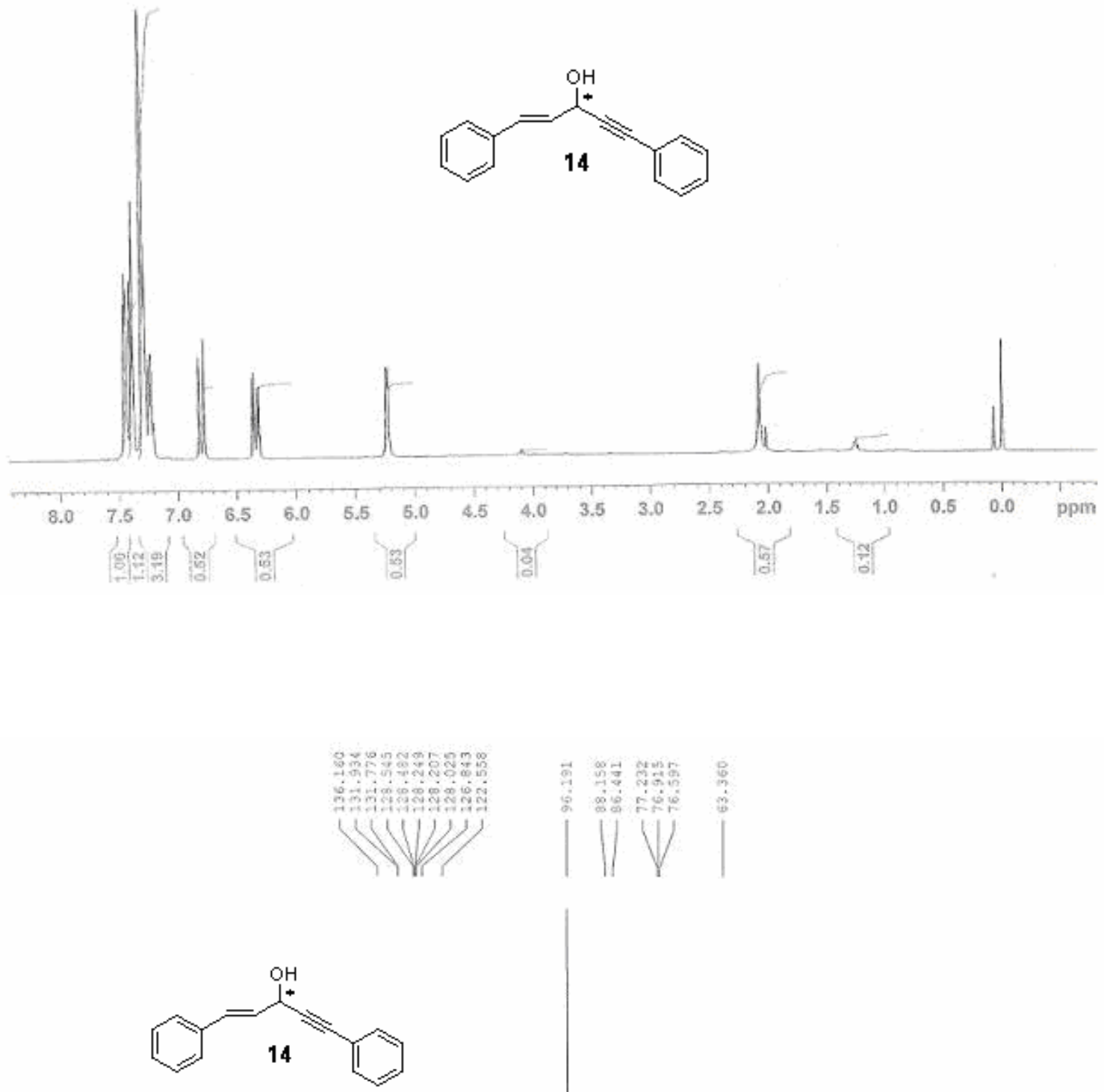

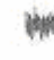

$\begin{array}{lllllllllllllllllllllll}210 & 200 & 190 & 180 & 170 & 160 & 150 & 140 & 130 & 120 & 110 & 100 & 90 & 80 & 70 & 60 & 50 & 40 & 30 & 20 & 10 & 0 & \text { ppm }\end{array}$ 

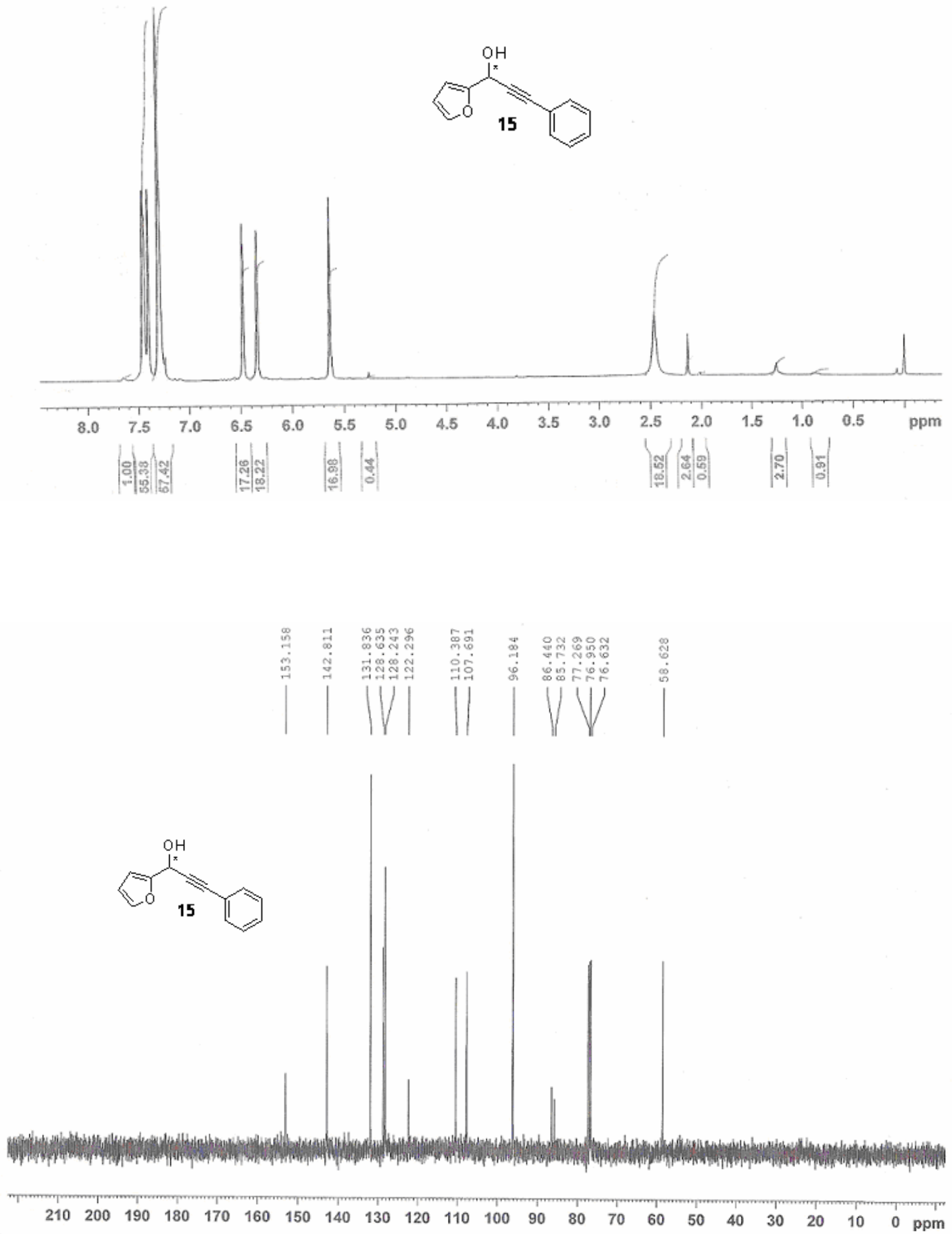

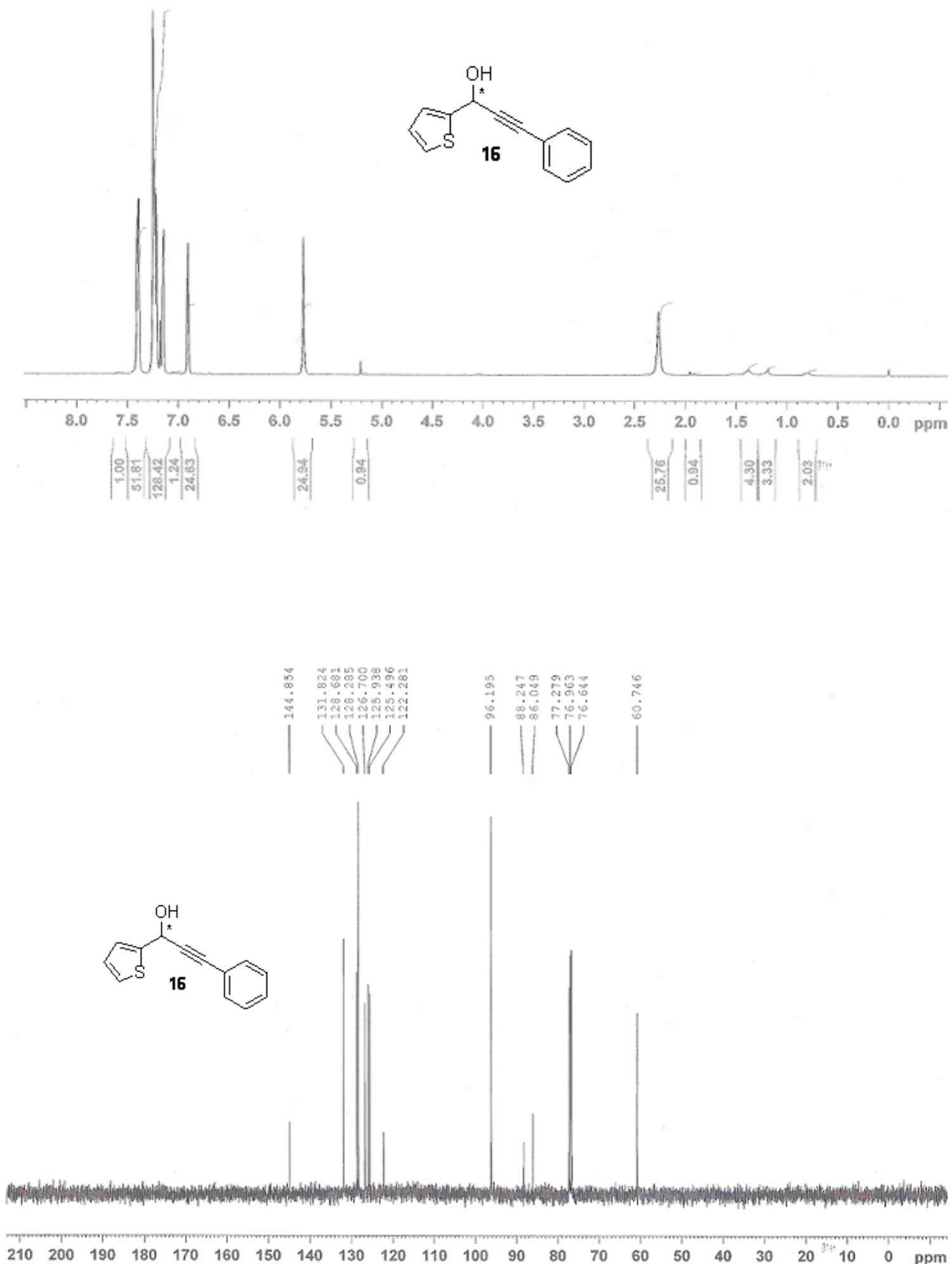

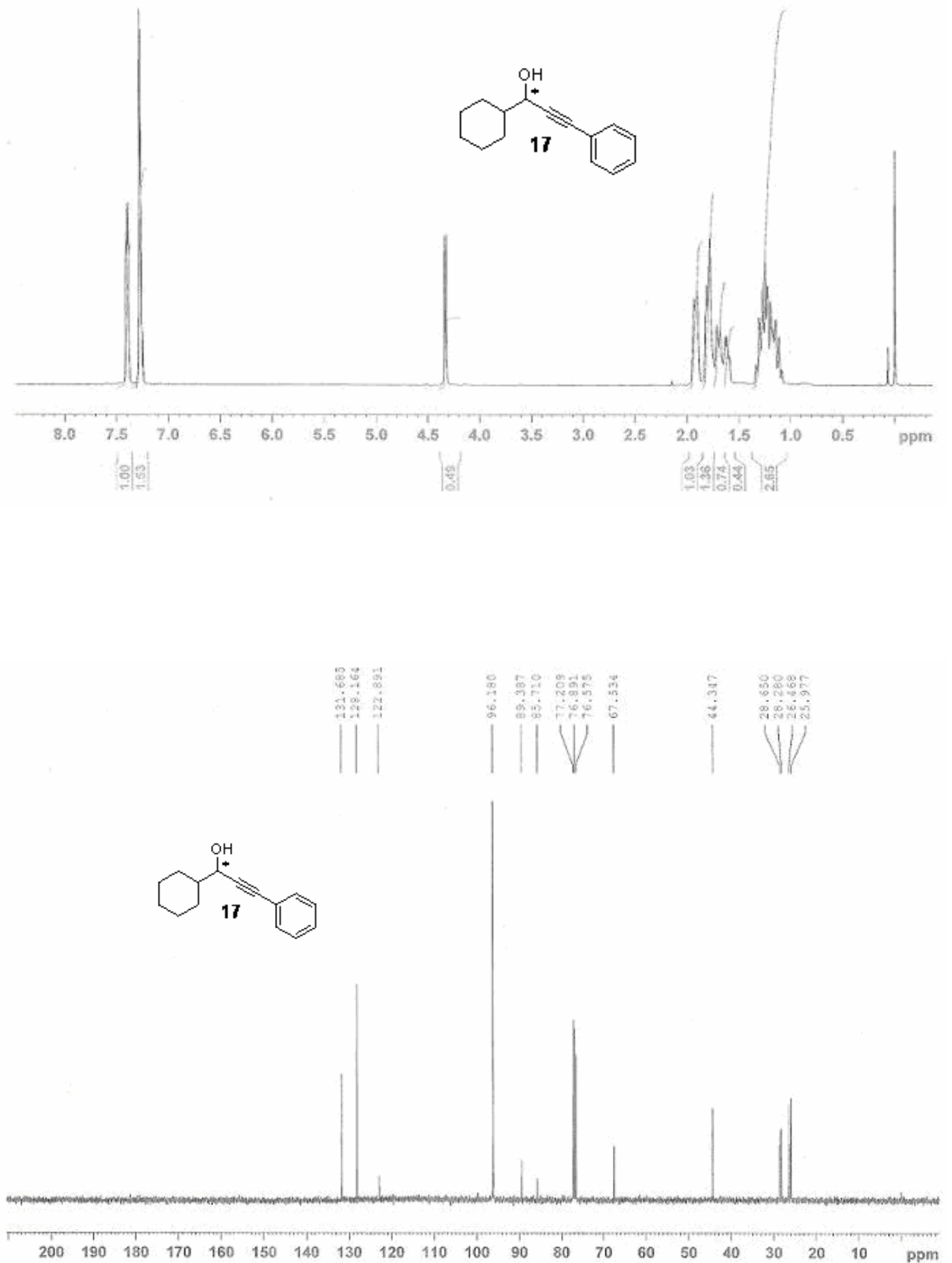

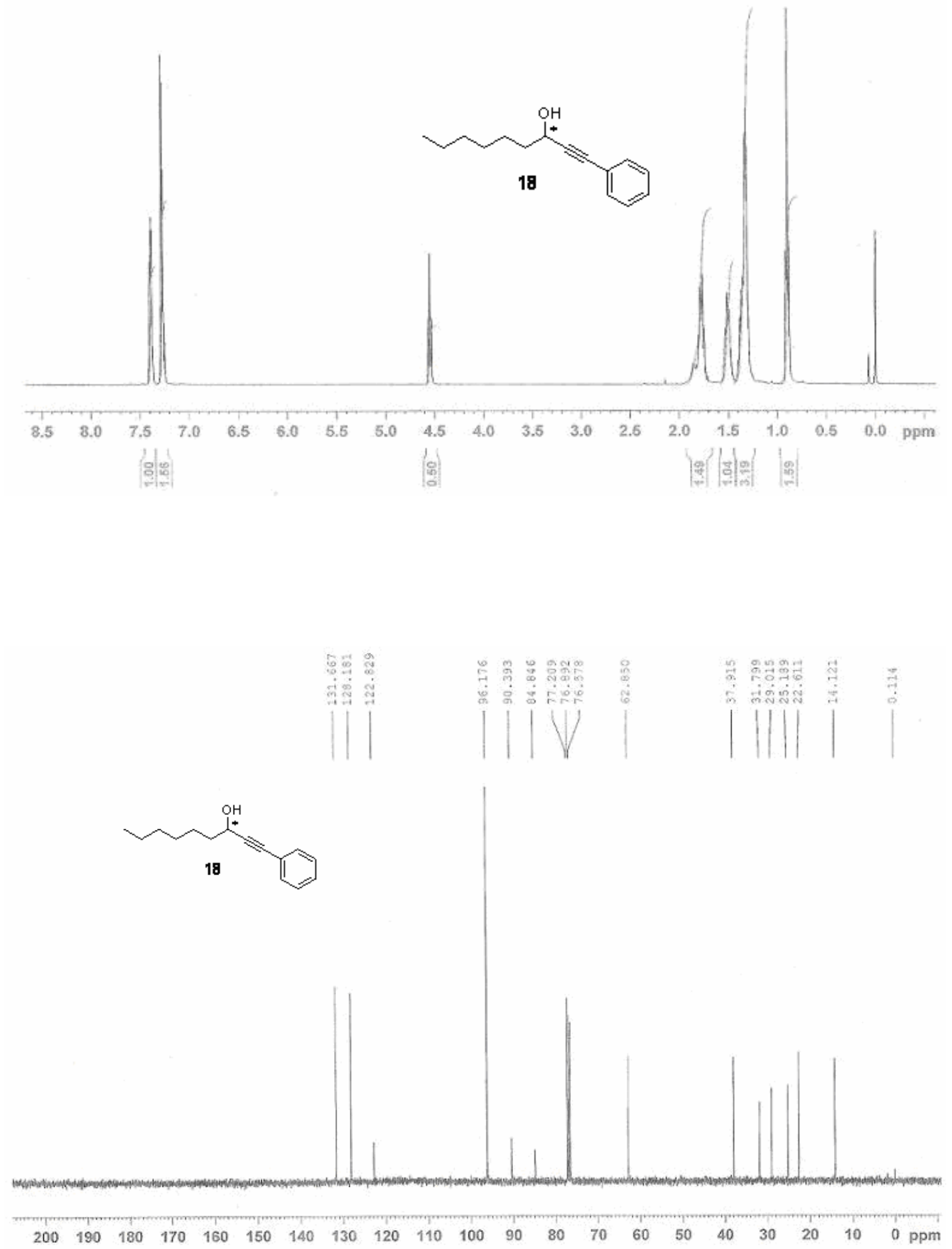

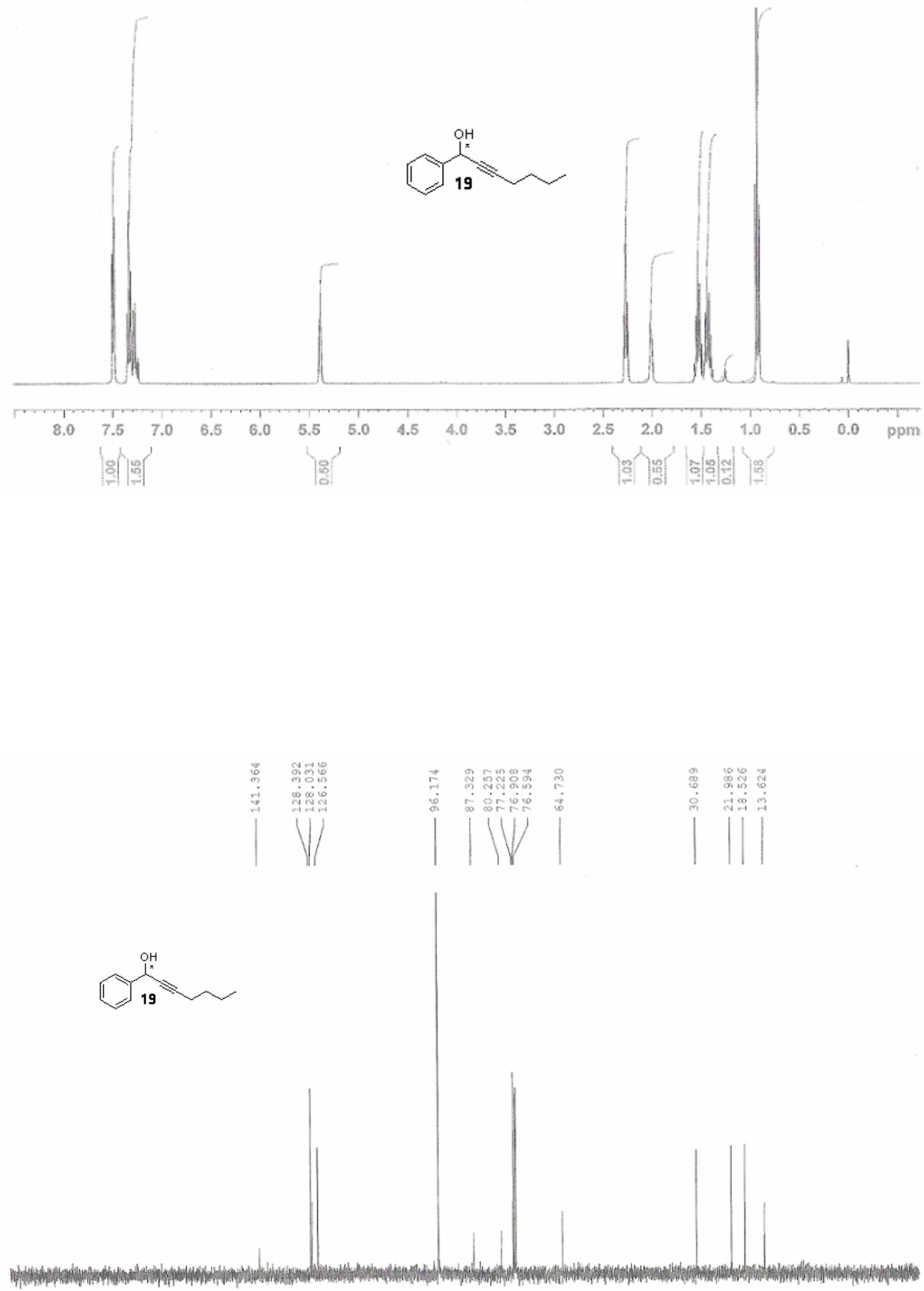

$\begin{array}{lllllllllllllllllllllll}200 & 190 & 180 & 170 & 160 & 150 & 140 & 130 & 120 & 110 & 100 & 90 & 80 & 70 & 60 & 50 & 40 & 30 & 20 & 10 & 0 & -10 & \text { ppm }\end{array}$ 

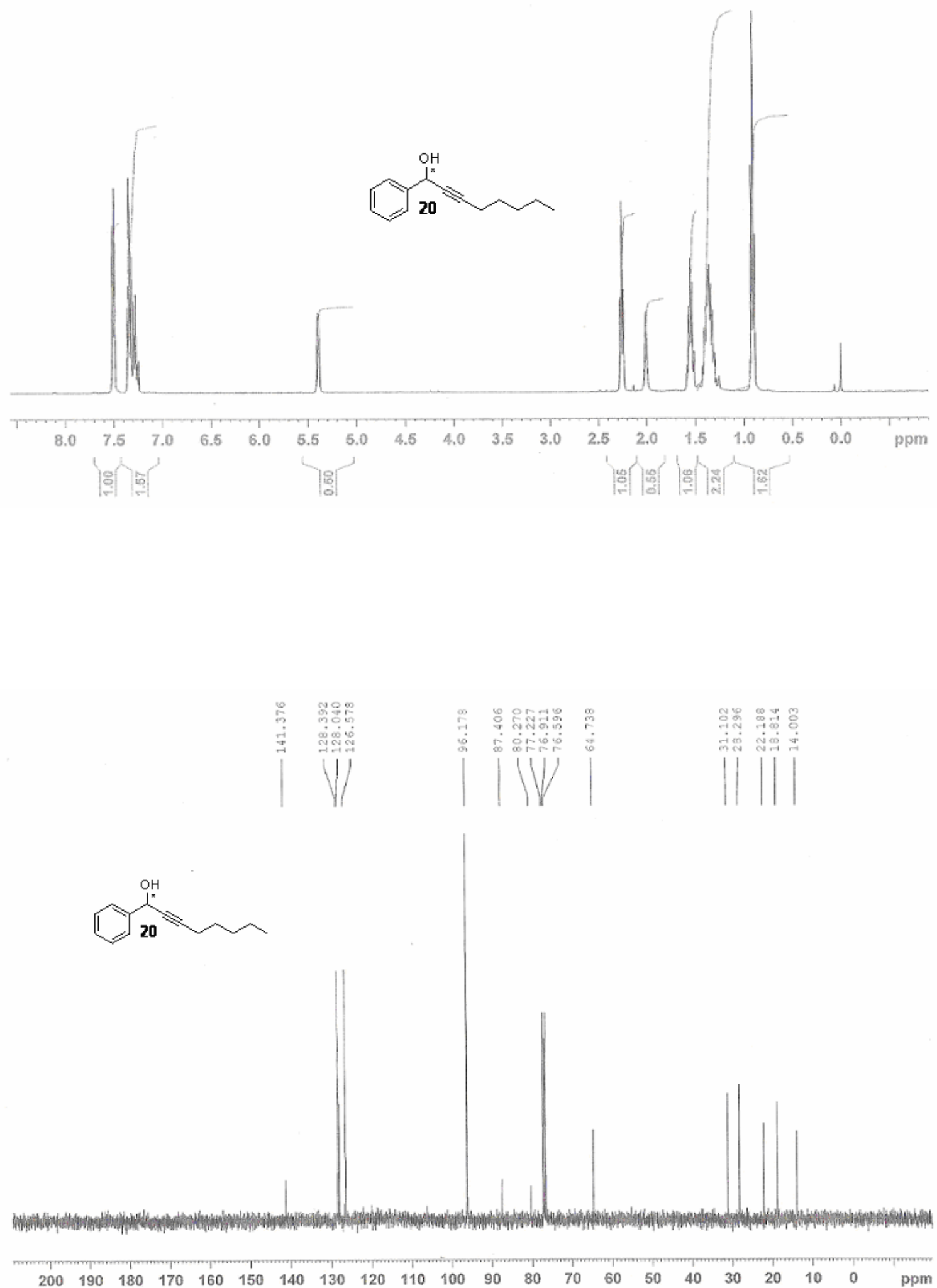
489 DKH459B2

\begin{tabular}{llll}
\hline Samplo Name: & DKH459B2 & Injection Volume: & $\mathbf{2 0 , 0}$ \\
Vial Number: & 501 & Channel: & UV_VIS_3 \\
Sample Type: & unknown & Wavelength: & $\mathbf{2 5 4}$ \\
Control Program: & PROGRAM01 & Bandwidth: & $\mathbf{2}$ \\
Quantif. Method: & METHOD01 & Divition Factor: & 1,0000 \\
Recording Time: & $\mathbf{2 3 . 2 . 2 0 0 7 ~ 2 1 : 5 6}$ & Sample Weight: & $\mathbf{1 , 0 0 0 0}$ \\
Run Time (min): & $\mathbf{2 6 , 0 3}$ & Sample Amount: & $\mathbf{1 , 0 0 0 0}$ \\
\hline
\end{tabular}

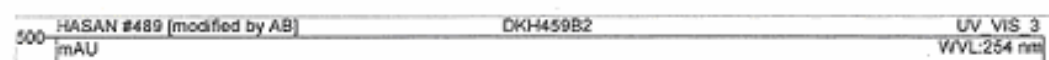

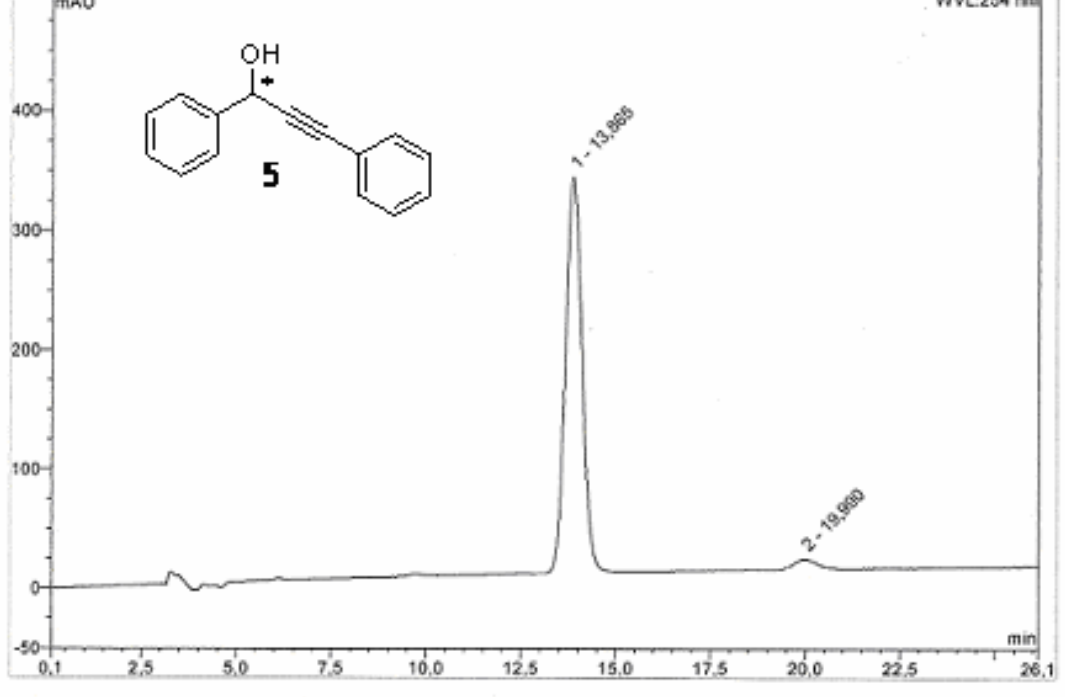

\begin{tabular}{|c|c|c|c|c|c|c|c|}
\hline No. & $\begin{array}{c}\text { Ret.Time } \\
\text { min }\end{array}$ & Peak Name & $\begin{array}{c}\text { Height } \\
\text { mAU }\end{array}$ & $\begin{array}{c}\text { Area } \\
\text { mAU*min }\end{array}$ & $\begin{array}{c}\text { Rel.Area } \\
\%\end{array}$ & Amount & Type \\
\hline 1 & 13,86 & n.a. & 331,137 & 169,128 & 97,75 & n.a. & $\mathrm{BM}^{*}$ \\
\hline 2 & 19.99 & n.a. & 6.839 & 3.888 & 2.25 & n.a. & $\mathrm{BMB}^{*}$ \\
\hline Total: & & & 337,976 & 173,015 & 100,00 & 0,000 & \\
\hline
\end{tabular}




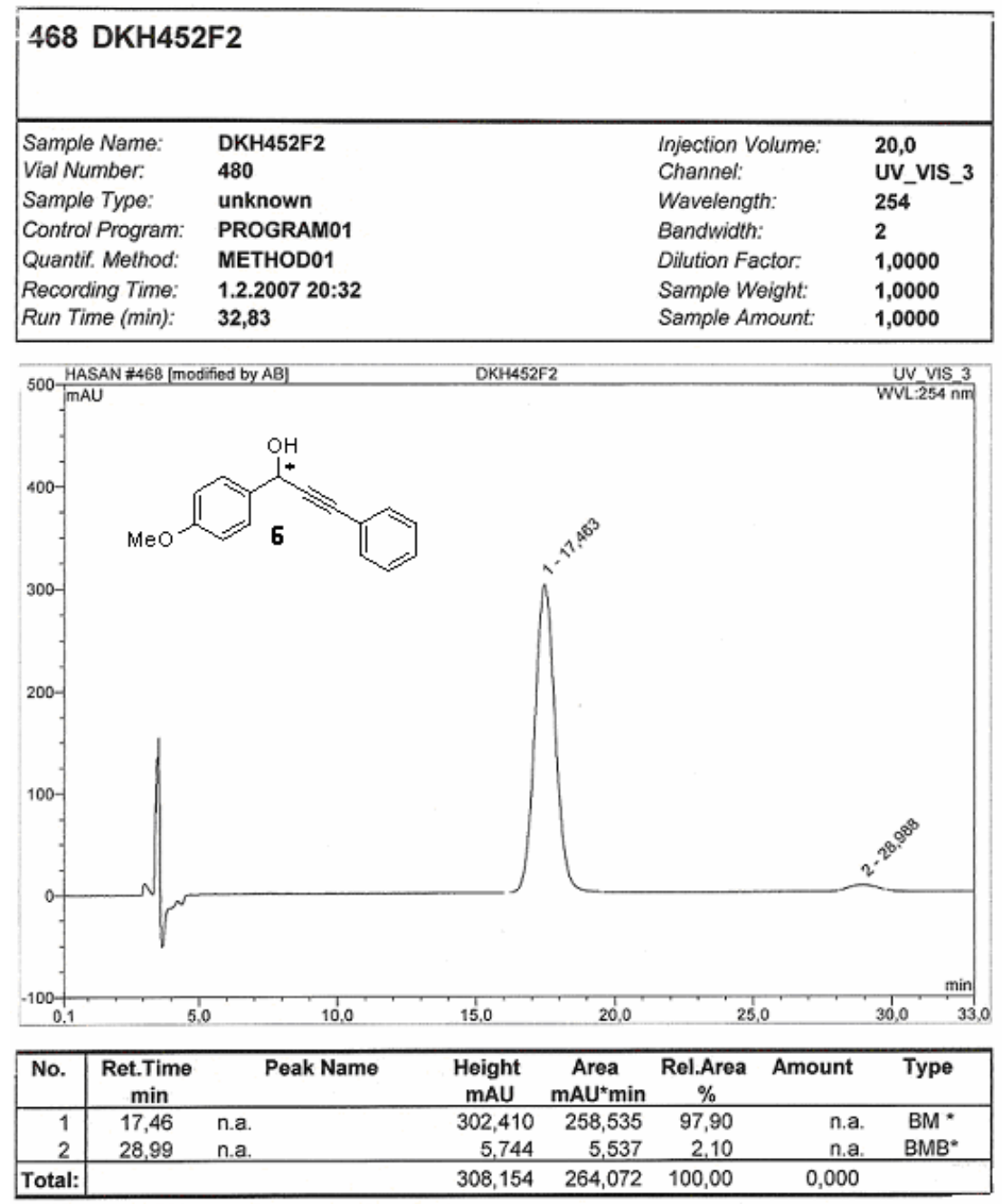




\section{DKH448C2}

\begin{tabular}{llll|}
\hline Sample Name: & DKH448C2 & Injection Volume: & $\mathbf{2 0 , 0}$ \\
Vial Number: & $\mathbf{4 6 9}$ & Channel: & UV_VIS_3 \\
Sample Type: & unknown & Wavelength: & $\mathbf{2 5 4}$ \\
Cantrol Program: & PROGRAM01 & Bandwidth: & $\mathbf{2}$ \\
Quantif. Method: & METHOD01 & Dilution Factor: & $\mathbf{1 , 0 0 0 0}$ \\
Recording Time: & $\mathbf{1 7 . 1 . 2 0 0 7 ~ 1 8 : 3 1}$ & Sample Woight: & $\mathbf{1 , 0 0 0 0}$ \\
Run Time (min): & $\mathbf{2 4 , 8 8}$ & Sample Amount: & $\mathbf{1 , 0 0 0 0}$ \\
\hline
\end{tabular}

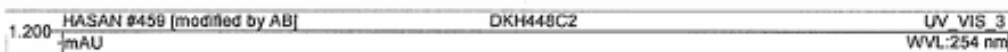

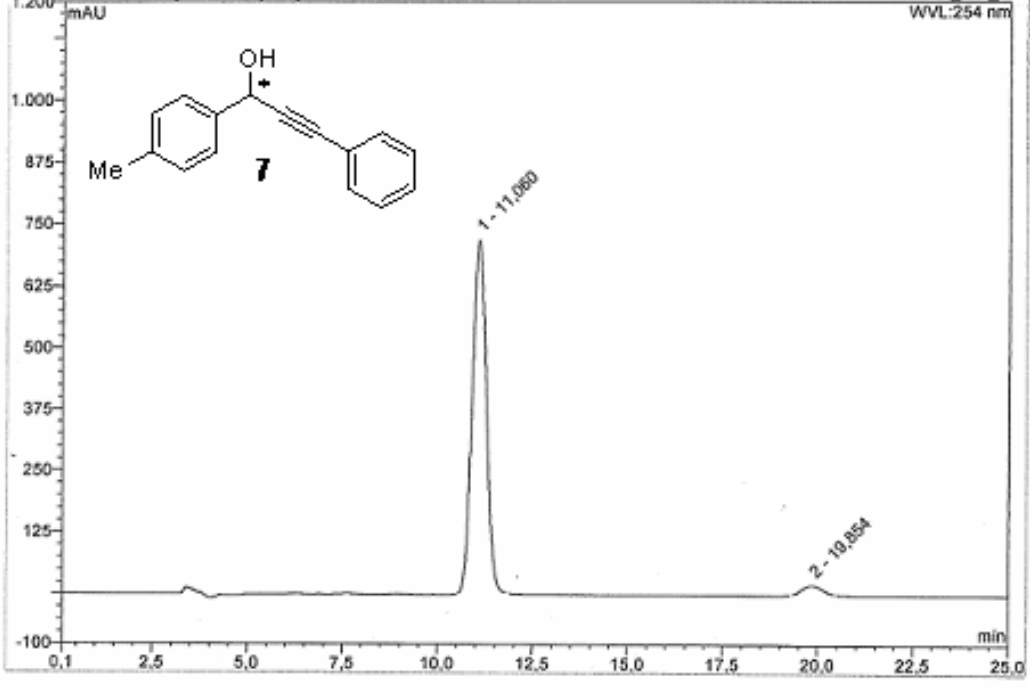

\begin{tabular}{|r|ccrrrrr|}
\hline No. & $\begin{array}{c}\text { Ret.Time } \\
\text { min }\end{array}$ & Peak Name & $\begin{array}{c}\text { Height } \\
\text { mAU }\end{array}$ & $\begin{array}{r}\text { Area } \\
\text { mAU*min }\end{array}$ & $\begin{array}{r}\text { Rel_Area } \\
\%\end{array}$ & Amount & Type \\
\hline 1 & 11,06 & n.a. & 715,563 & 306,841 & 96,40 & n.a. & BMB* $^{*}$ \\
2 & 19,85 & n.a. & 18,720 & 11,469 & 3,60 & n.a. & BMB $^{*}$ \\
\hline Total: & & & 734,282 & 318,309 & 100,00 & 0,000 & \\
\hline
\end{tabular}


440 DKH439D1

Sample Name:

Vial Number:

Sample Type:

Control Program:

Quantif. Mothod

Recording Time:

Run Time (min):

DKH439D1

450

unknown

PROGRAM01

METHOD01

28.12.2006 23:45

54,82
Injection Vofume:

Channel:

Wavelength:

Bandwioth:

Dilution Factor:

Sample Weight:

Sample Amount:
20,0

UV_VIS_3

254

2

1,0000

1,0000

1,0000

\begin{tabular}{|c|c|c|}
\hline 2500 HASAN $A 440$ [modified by $A B]$ & DKH43801 & UV YIS 3 \\
\hline
\end{tabular}

$2.500-\mathrm{mat}$<smiles>O[C@H](C#Cc1ccccc1)c1ccc(Cl)cc1</smiles>

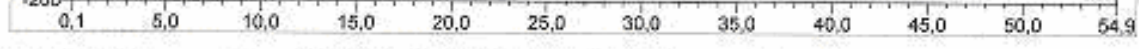

\begin{tabular}{|c|c|c|c|c|c|c|c|}
\hline No. & $\begin{array}{c}\text { Ret.Time } \\
\text { min }\end{array}$ & Peak Name & $\begin{array}{c}\text { Height } \\
\text { mAU }\end{array}$ & $\begin{array}{c}\text { Area } \\
\text { mAU*min }\end{array}$ & $\begin{array}{c}\text { Rel.Area } \\
\%\end{array}$ & Amount & Type \\
\hline 1 & 14,82 & n.a. & 1618,131 & 907,709 & 97,27 & n.a. & BMB $^{*}$ \\
\hline 2 & 47.79 & n.a. & 20.963 & 25,481 & 2,73 & n.a. & $\mathrm{BMB}^{*}$ \\
\hline Total: & & & 1639,094 & 933,189 & 100,00 & 0,000 & \\
\hline
\end{tabular}




\section{DKH441C2}

\begin{tabular}{|llll|}
\hline Sample Name: & DKH441C2 & Injection Volume: & $\mathbf{2 0 , 0}$ \\
Vial Number: & $\mathbf{4 5 5}$ & Channel: & UV_VIS_3 \\
Sample Type: & unknown & Wavelength: & 254 \\
Control Program: & PROGRAM01 & Bandwidth: & 2 \\
Quantif. Method: & METHOD01 & Dilution Factor: & 1,0000 \\
Recording Time: & $\mathbf{2 9 . 1 2 . 2 0 0 6 3 : 4 6}$ & Sample Weight: & 1,0000 \\
Run Time (min): & $\mathbf{5 6 , 8 0}$ & Sample Amount: & $\mathbf{1 , 0 0 0 0}$ \\
\hline
\end{tabular}
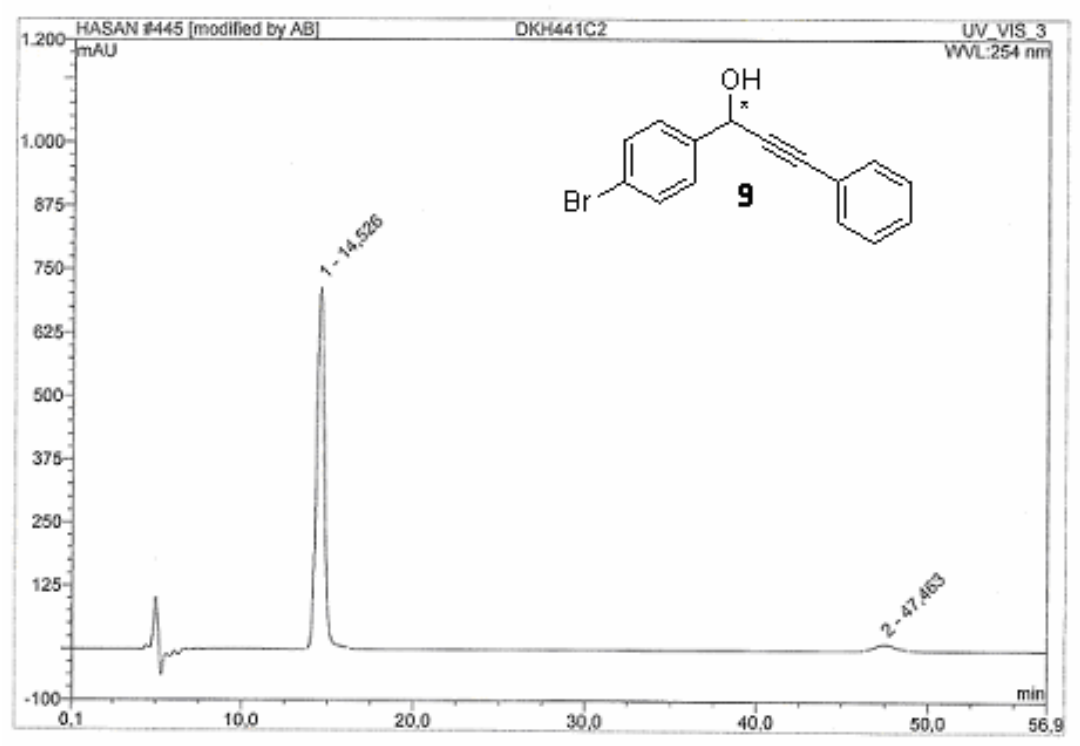

\begin{tabular}{|r|rrrrrrr|}
\hline No. & $\begin{array}{c}\text { Ret.Time } \\
\text { min }\end{array}$ & Peak Name & $\begin{array}{c}\text { Height } \\
\text { mAU }\end{array}$ & $\begin{array}{c}\text { Area } \\
\text { mAU*min }\end{array}$ & $\begin{array}{c}\text { Rel.Area } \\
\%\end{array}$ & Amount & Type \\
\hline 1 & 14,53 & n.a. & 711,981 & 377,871 & 96,10 & n.a. & BMB $^{*}$ \\
2 & 47,46 & n.a. & 11,001 & 15,341 & 3,90 & n.a. & BMB $^{*}$ \\
\hline Total: & & & 722,982 & 393,212 & 100,00 & 0,000 & \\
\hline
\end{tabular}




\section{DKH438C2}

\begin{tabular}{llll}
\hline Sample Name: & DKH438C2 & injection Volume: & 20,0 \\
Vial Number: & 449 & Channel: & 254 \\
Sample Type: & unknown & Wavelength: & 2 \\
Control Program: & PROGRAM01 & Bandwidth: & 2 \\
Quantif. Method: & METHOD01 & Dilution Factor: & 1,0000 \\
Recording Time: & $26.12 .200611: 52$ & Sample Weight: & 1,0000 \\
Run Time (min): & 50,54 & Sample Amount: & 1,0000
\end{tabular}

50,54

UV_VIS 3

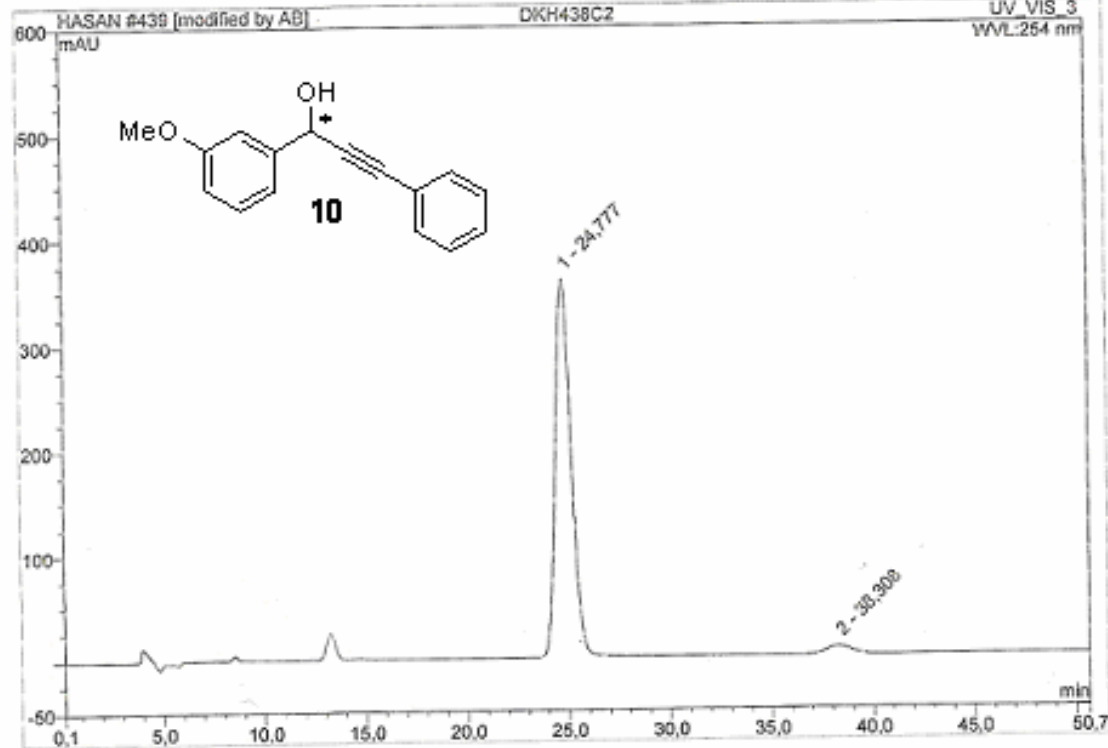

\begin{tabular}{|r|ccrrrrr|}
\hline No. & $\begin{array}{r}\text { Ret.Time } \\
\text { min }\end{array}$ & Peak Name & $\begin{array}{c}\text { Height } \\
\text { mAU }\end{array}$ & $\begin{array}{c}\text { Area } \\
\text { mAU'min }\end{array}$ & $\begin{array}{r}\text { Rel.Area } \\
\%\end{array}$ & Amount & Type \\
\hline 1 & 24,78 & n.a. & 358,815 & 321,079 & 97,55 & n.a. & BMB $^{*}$ \\
2 & 38,31 & n.a. & 6,736 & 8,047 & 2,45 & n.a. & BMB $^{*}$ \\
\hline Total: & & & 365,551 & 329,126 & 100,00 & 0,000 & \\
\hline
\end{tabular}




\begin{tabular}{|llll|}
\hline 447 DKH442D2 & & \\
& & & \\
\hline Sample Name: & DKH442D2 & Injection Volume: & 20,0 \\
Vial Number: & 457 & Channel: & UV_VIS_3 \\
Sample Type: & unknown & Wavelength: & 254 \\
Control Program: & PROGRAM01 & Bandwidth: & 2 \\
Quantif. Method: & METHOD01 & Dilution Factor: & $\mathbf{1 , 0 0 0 0}$ \\
Recording Time: & 29.12 .2006 5:44 & Sample Weight: & 1,0000 \\
Run Time (min): & $\mathbf{5 9 , 8 8}$ & Sample Amount: & $\mathbf{1 , 0 0 0 0}$ \\
\hline
\end{tabular}

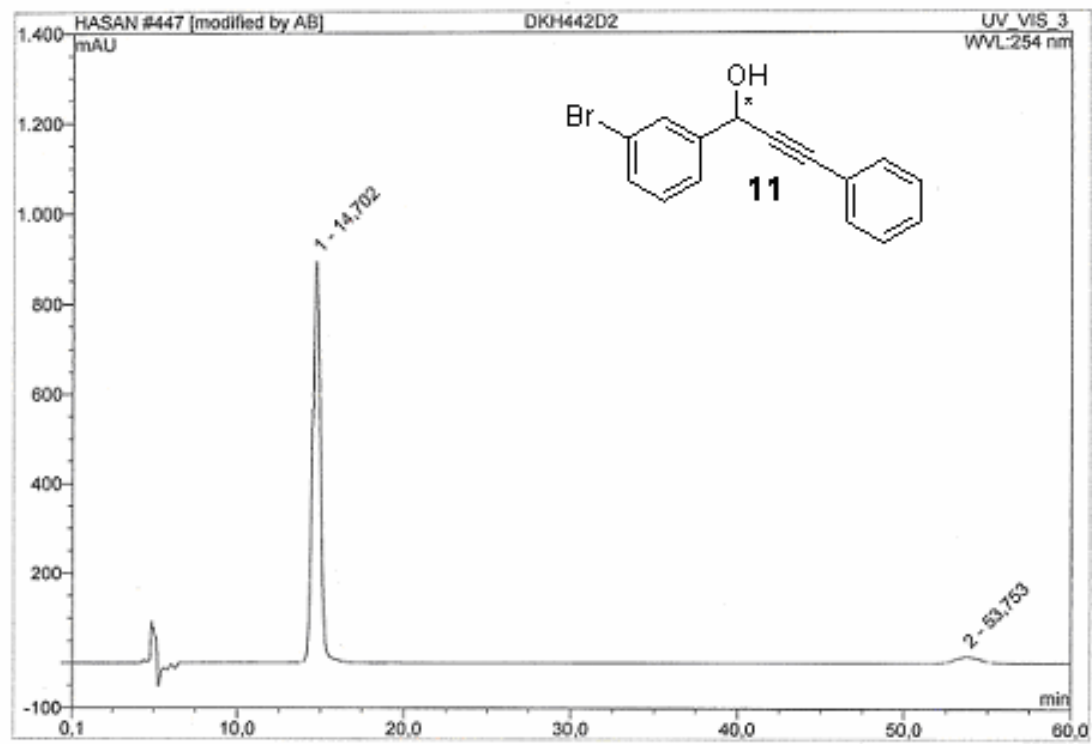

\begin{tabular}{|c|c|c|c|c|c|c|c|}
\hline No. & $\begin{array}{c}\text { Ret.Time } \\
\text { min }\end{array}$ & Peak Name & $\begin{array}{c}\text { Height } \\
\text { mAU }\end{array}$ & $\begin{array}{c}\text { Area } \\
\text { mAUmin }\end{array}$ & $\begin{array}{c}\text { Rel.Area } \\
\%\end{array}$ & Amount & Type \\
\hline 1 & 14,70 & ก.a. & 891,810 & 482,647 & 96,05 & n.a. & BMB* \\
\hline 2 & 53,75 & n.a. & 12,868 & 19,858 & 3,95 & n.a. & $\mathrm{BMB}^{*}$ \\
\hline Total: & & & 904,678 & 502,505 & 100,00 & 0,000 & \\
\hline
\end{tabular}




\begin{tabular}{|llll|}
\hline 414 DKH429C2 & & \\
& & & \\
\hline Sample Name: & DKH429C2 & injection Volume: & 20,0 \\
Vial Number: & 423 & Channet: & UV_VIS_3 \\
Sample Type: & unknown & Wavelength: & 254 \\
Control Program: & PROGRAM01 & Bandwidth: & 2 \\
Quantif. Method: & METHOD01 & Dilution Factor: & $\mathbf{1 , 0 0 0 0}$ \\
Recording Time: & 11.12 .2006 5:27 & Sample Weight: & $\mathbf{1 , 0 0 0 0}$ \\
Run Timo (min): & 34,84 & Sample Amount: & $\mathbf{1 , 0 0 0 0}$ \\
\hline
\end{tabular}
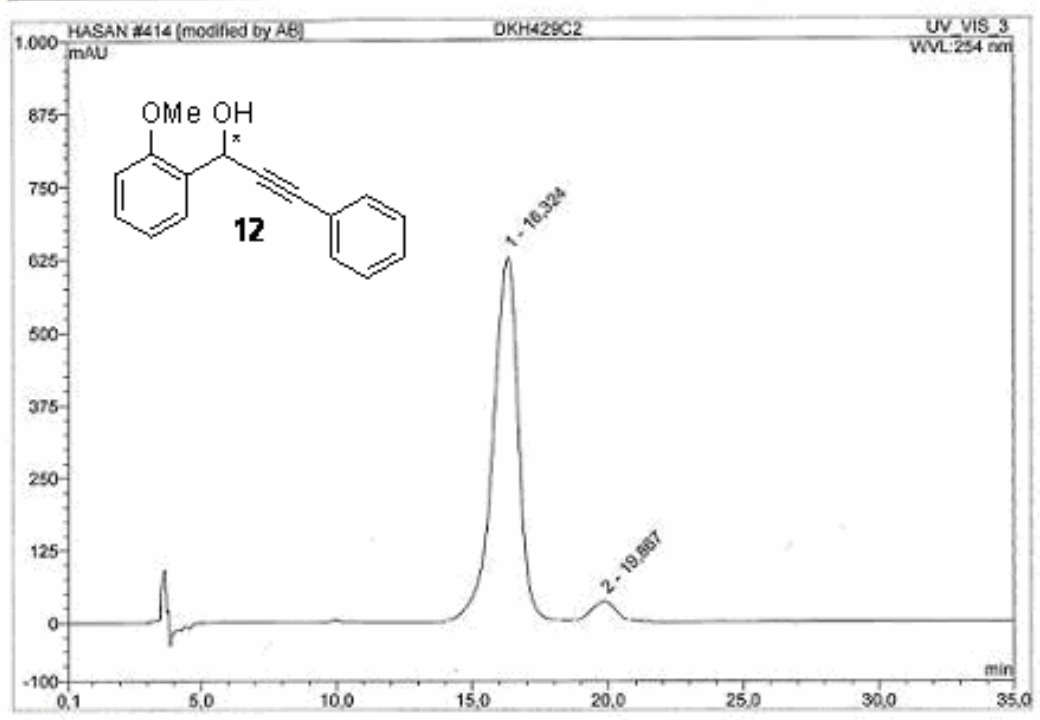

\begin{tabular}{|r|rrrrrrr|}
\hline No. & $\begin{array}{c}\text { Ret. Time } \\
\text { min }\end{array}$ & Peak Name & $\begin{array}{c}\text { Height } \\
\text { mAU }\end{array}$ & $\begin{array}{c}\text { Area } \\
\text { mAU'min }\end{array}$ & $\begin{array}{r}\text { Rel.Area } \\
\%\end{array}$ & Amount & Type \\
\hline 1 & 16,32 & n.a. & 623,648 & 672,205 & 95,59 & n.a. & BMB* $^{*}$ \\
2 & 19,87 & n.a. & 31,691 & 31,042 & 4,41 & n.a. & BMB* $^{*}$ \\
\hline Total: & & & 655,339 & 703,247 & 100,00 & 0,000 & \\
\hline
\end{tabular}


470 DKH453C2

\begin{tabular}{llll|}
\hline Sample Name: & DKH453C2 & Injection Volume: & 20,0 \\
Vial Number: & 482 & Channel: & UV_VIS_3 \\
Sample Type: & unknown & Wavelength: & 254 \\
Control Program: & PROGRAM01 & Bandwidth: & 2 \\
Quantif, Method: & METHOD01 & Dilution Factor: & 1,0000 \\
Recording Time: & $1.2 .200722: 02$ & Sample Weight: & 1,0000 \\
Run Time $(m i n):$ & 54,83 & Sample Amount: & 1,0000 \\
\hline
\end{tabular}

400 MASAN EA7O [modifiad by AB] OKHA53C2 UV VIS 3

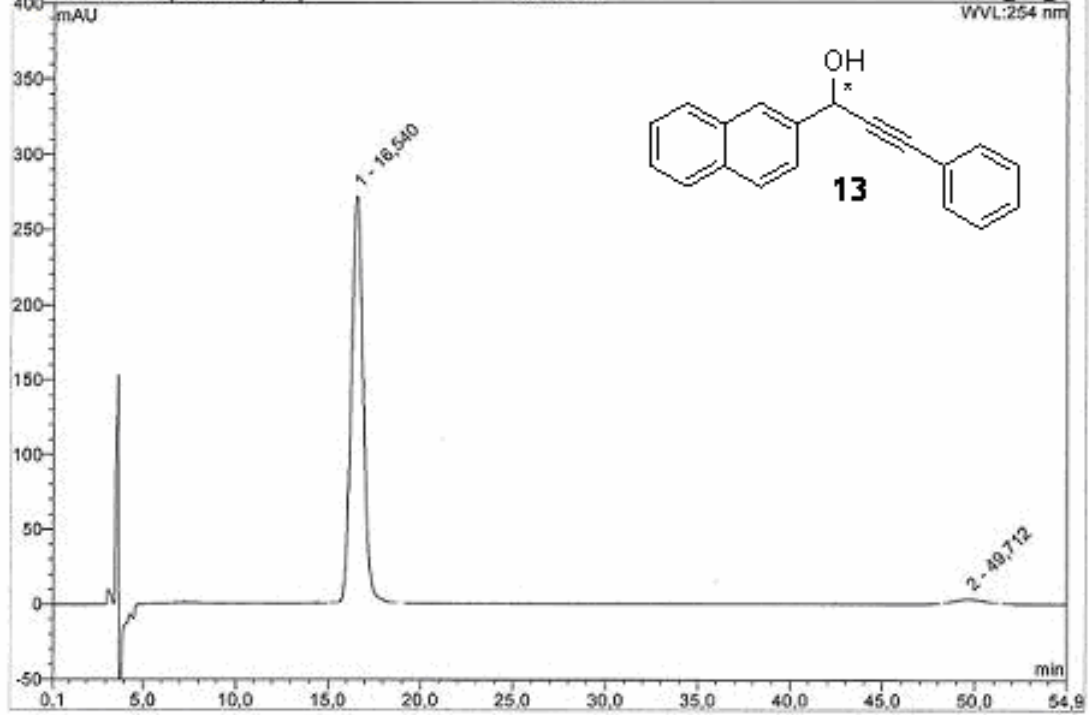

\begin{tabular}{|c|c|c|c|c|c|c|c|}
\hline No. & $\begin{array}{c}\text { Ret.Time } \\
\text { min }\end{array}$ & Peak Name & $\begin{array}{c}\text { Height } \\
\text { mAU }\end{array}$ & $\begin{array}{c}\text { Area } \\
\text { mAU'min }\end{array}$ & $\begin{array}{c}\text { Rel.Area } \\
\%\end{array}$ & Amount & Type \\
\hline 1 & 16,54 & n.a. & 271,035 & 204,438 & 97,61 & n.a. & $\mathrm{BMB}^{*}$ \\
\hline 2 & 49,71 & n.a. & 2,875 & 5,010 & 2,39 & n.a. & BMB* \\
\hline Total: & & & 273,910 & 209,448 & 100,00 & 0,000 & \\
\hline
\end{tabular}




\begin{tabular}{|llll|}
\hline 448 DKH443C1 & & \\
\hline Sample Name: & DKH443C1 & & \\
\hline Vial Number: & 458 & Injection Volume: & 20,0 \\
Sample Type: & unknown & Channel: & UV_VIS_3 \\
Control Program: & PROGRAM01 & Wavelength: & $\mathbf{2 5 4}$ \\
Quantif. Method: & METHOD01 & Bandwidth: & $\mathbf{2}$ \\
Recording Time: & $\mathbf{9 . 1 . 2 0 0 7 ~ 1 2 : 1 1 ~}$ & Dilution Factor: & $\mathbf{1 , 0 0 0 0}$ \\
Run Time (min): & $\mathbf{7 9 , 8 8}$ & Sample Weight: & $\mathbf{1 , 0 0 0 0}$ \\
\hline
\end{tabular}

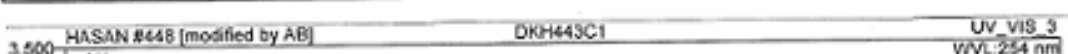

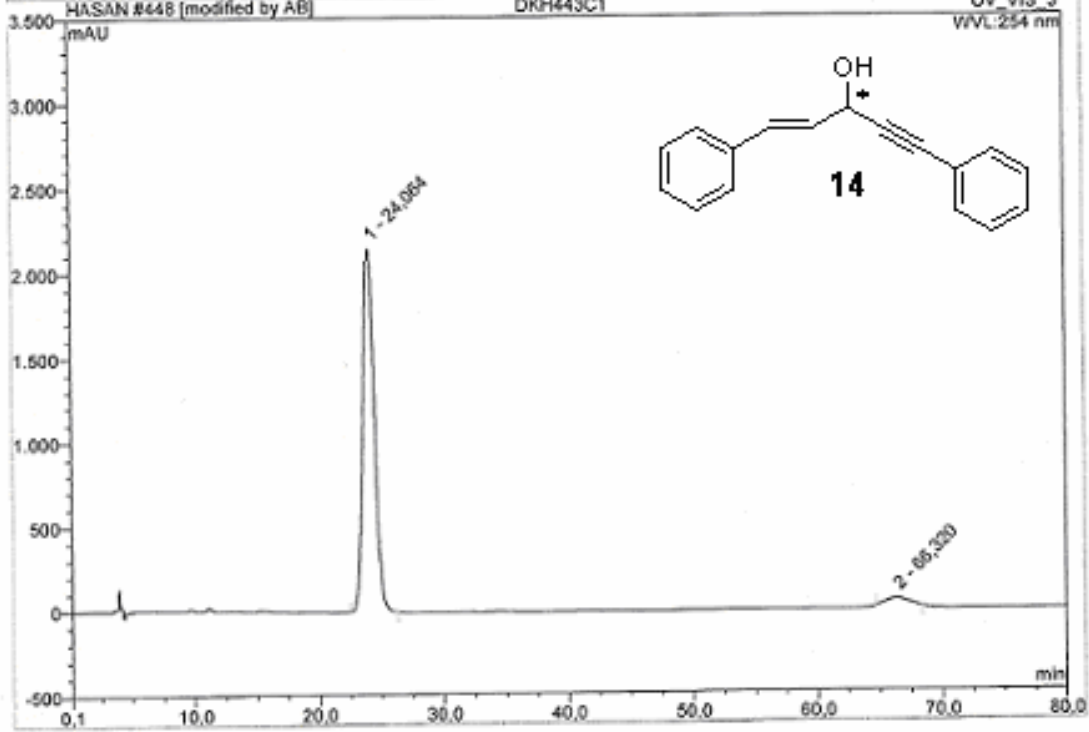

\begin{tabular}{|r|ccrrrrr|}
\hline No. & $\begin{array}{c}\text { Ret.Time } \\
\text { min }\end{array}$ & Peak Name & $\begin{array}{c}\text { Height } \\
\text { mAU }\end{array}$ & $\begin{array}{c}\text { Area } \\
\text { mAU*min }\end{array}$ & $\begin{array}{r}\text { Rel.Area } \\
\%\end{array}$ & Amount & Type \\
\hline 1 & 24,06 & n.a. & 2146,602 & 2298,192 & 96,43 & n.a. & BMB $^{*}$ \\
2 & 66,32 & n.a. & 40,358 & 84,960 & 3,57 & n.a. & BMB $^{*}$ \\
\hline Total: & & & 2186,959 & 2383,152 & 100,00 & 0,000 & \\
\hline
\end{tabular}




\section{DKH437C2}

\begin{tabular}{llll|}
\hline Sample Name: & DKH437C2 & Injection Volume: & $\mathbf{2 0 , 0}$ \\
Vial Number: & 447 & Channel: & UV_VIS_3 \\
Sample Type: & unknown & Wavolength: & $\mathbf{2 5 4}$ \\
Control Program: & PROGRAM01 & Bandwidth: & $\mathbf{2}$ \\
Quantif. Method: & METHOD01 & Dilution Factor: & $\mathbf{1 , 0 0 0 0}$ \\
Recording Time: & $\mathbf{2 6 . 1 2 . 2 0 0 6 ~ 9 : 4 7}$ & Sample Weight: & $\mathbf{1 , 0 0 0 0}$ \\
Run Time (min): & $\mathbf{4 2 , 4 5}$ & Sample Amount: & 1,0000 \\
\hline
\end{tabular}

600 HASAN 2437 (modfied by AB] OKH437C2 $\quad$ UV VIS_3

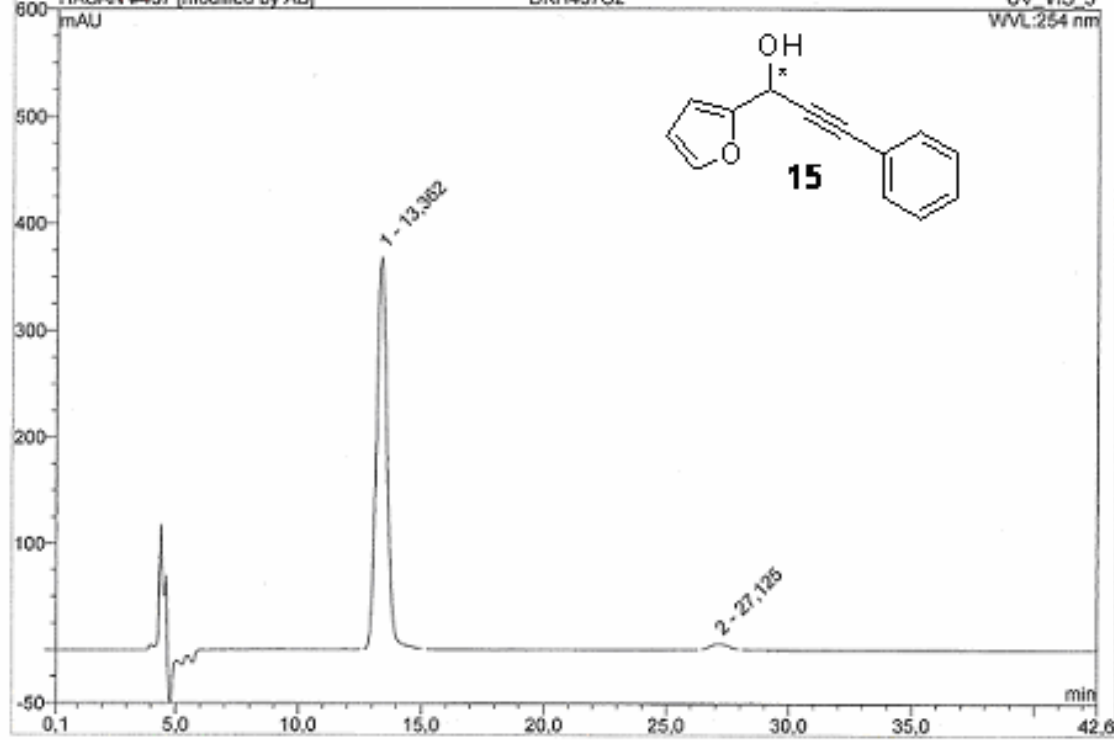

\begin{tabular}{|c|c|c|c|c|c|c|c|}
\hline No. & $\begin{array}{c}\text { Ret.Time } \\
\text { min }\end{array}$ & Peak Name & $\begin{array}{c}\text { Height } \\
\text { mAU }\end{array}$ & $\begin{array}{c}\text { Area } \\
\text { mAU*min }\end{array}$ & $\begin{array}{c}\text { Rel.Area } \\
\%\end{array}$ & Amount & Type \\
\hline 1 & 13,36 & n.a. & 366,898 & 184,758 & 97,67 & n.a. & $\mathrm{BMB}^{*}$ \\
\hline 2 & 27.13 & n.a. & 5,151 & 4,404 & 2,33 & n.a. & $\mathrm{BMB}^{*}$ \\
\hline Total: & & & 372,049 & 189,162 & 100,00 & 0,000 & \\
\hline
\end{tabular}




\section{DKH481B2}

\begin{tabular}{llll|}
\hline Sample Name: & DKH481B2 & Injection Volume: & 20,0 \\
Vial Number: & 548 & Channel: & UV_VIS_3 \\
Sample Type: & unknown & Wavelength: & 254 \\
Control Program: & PROGRAM01 & Bandwidlh: & 2 \\
Quant:. Method: & METHOD01 & Dilution Factor: & 1,0000 \\
Recording Time: & $30.3 .200713: 45$ & Sample Weight: & 1,0000 \\
Run Time (min): & 29,88 & Sample Amount: & 1,0000 \\
\hline
\end{tabular}

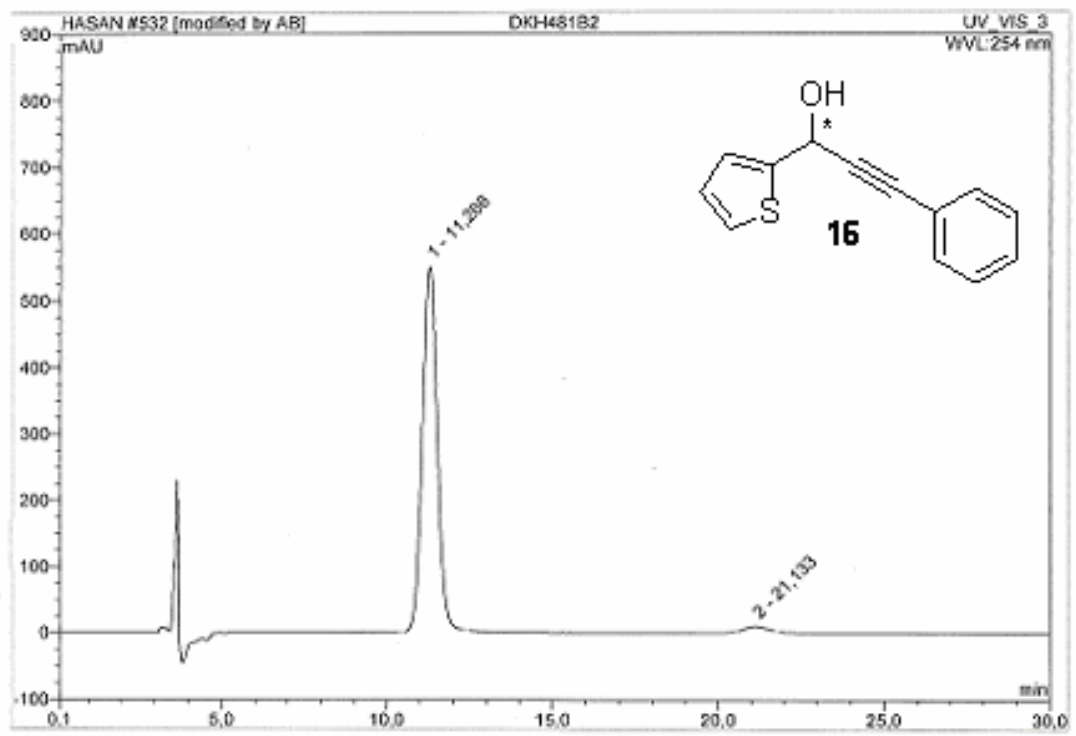

\begin{tabular}{|c|c|c|c|c|c|c|c|}
\hline No. & $\begin{array}{l}\text { Ret.Time } \\
\text { min }\end{array}$ & Peak Name & $\begin{array}{c}\text { Height } \\
\text { mAU }\end{array}$ & $\begin{array}{c}\text { Area } \\
\text { mAU'min }\end{array}$ & $\begin{array}{c}\text { Rel.Area } \\
\%\end{array}$ & Amount & Type \\
\hline 1 & 11,27 & n.a. & 548,512 & 283,242 & 98,21 & п.а. & $\mathrm{BMB}^{*}$ \\
\hline 2 & 21,13 & n.a. & 7,148 & 5,161 & 1,79 & n.a. & BMB: \\
\hline Total: & & & 555,660 & 288.403 & 100,00 & 0,000 & \\
\hline
\end{tabular}




\begin{tabular}{|llll|}
\hline 435 DKH435C2 & & \\
& & & \\
\hline Sample Name: & DKH435C2 & Injection Volume: & 20,0 \\
Vial Number: & 444 & Channel: & UV_VIS_3 \\
Sample Type: & unknown & Wavelength: & 254 \\
Control Program: & PROGRAM01 & Bandivith: & 2 \\
Quantif. Method: & METHOD01 & Silution Factor: & 1,0000 \\
Recording Time: & 26.12 .2006 6:31 & Sample Weight: & $\mathbf{1 , 0 0 0 0}$ \\
Run Time (min): & 24,83 & Sample Amount: & $\mathbf{1 , 0 0 0 0}$ \\
\hline
\end{tabular}

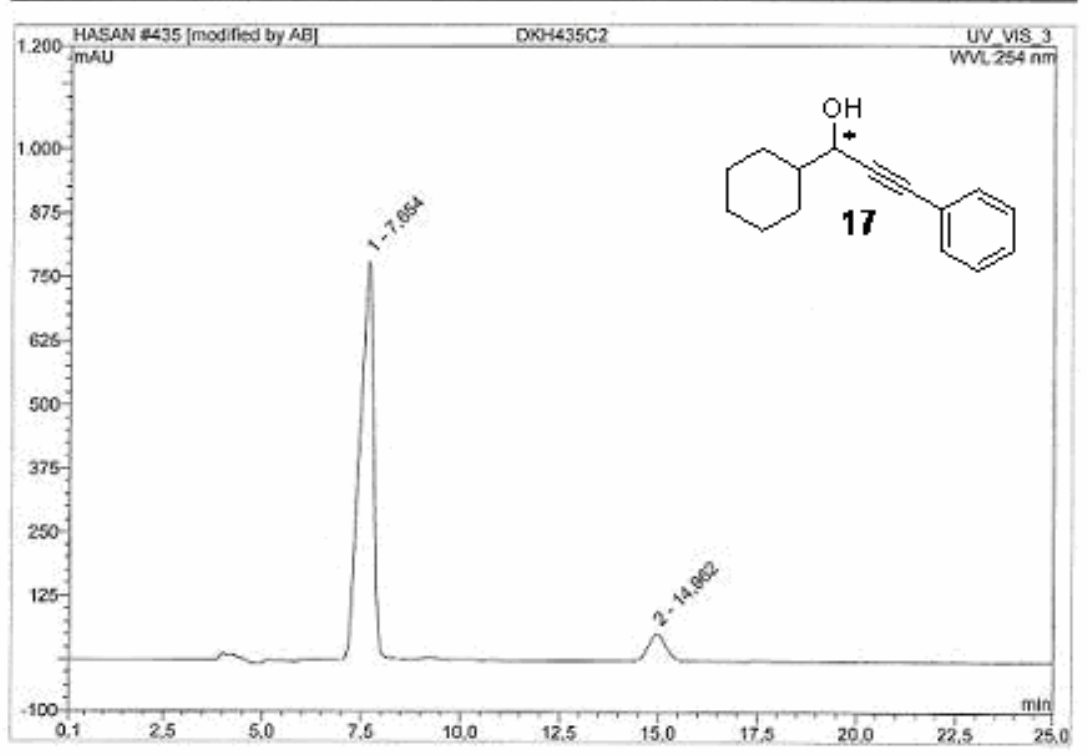

\begin{tabular}{|c|c|c|c|c|c|c|c|}
\hline No. & $\begin{array}{c}\text { Ret.Time } \\
\text { min }\end{array}$ & Peak Name & $\begin{array}{c}\text { Height } \\
\text { mAU }\end{array}$ & $\begin{array}{c}\text { Area } \\
\text { mAU*min }\end{array}$ & $\begin{array}{c}\text { Rel.Area } \\
\%\end{array}$ & Amount & Type \\
\hline 1 & 7.65 & n.a. & 777,205 & 311,420 & 92,68 & n.a. & $\mathrm{BMB}^{*}$ \\
\hline 2 & 14,96 & n.a. & 49,630 & 24,585 & 7,32 & n.a. & $\mathrm{BMB}^{*}$ \\
\hline Total: & & & 826,834 & 336,005 & 100,00 & 0,000 & \\
\hline
\end{tabular}




\section{DKH436C2}

\begin{tabular}{llll}
\hline Sample Name: & DKH436C2 & injection Volume: & 20,0 \\
Vial Number: & 442 & Channef: & UV_VIS_3 \\
Sample Type: & unknown & Wavelength: & 254 \\
Control Program: & PROGRAM01 & Bandwidth: & 2 \\
Quantif, Method: & METHOD01 & Dilution Factor: & 1,0000 \\
Recording Time: & $26.12 .20065: 39$ & Sample Weight: & 1,0000 \\
Run Time (min): & 24,81 & Sample Amount: & 1,0000 \\
\hline
\end{tabular}

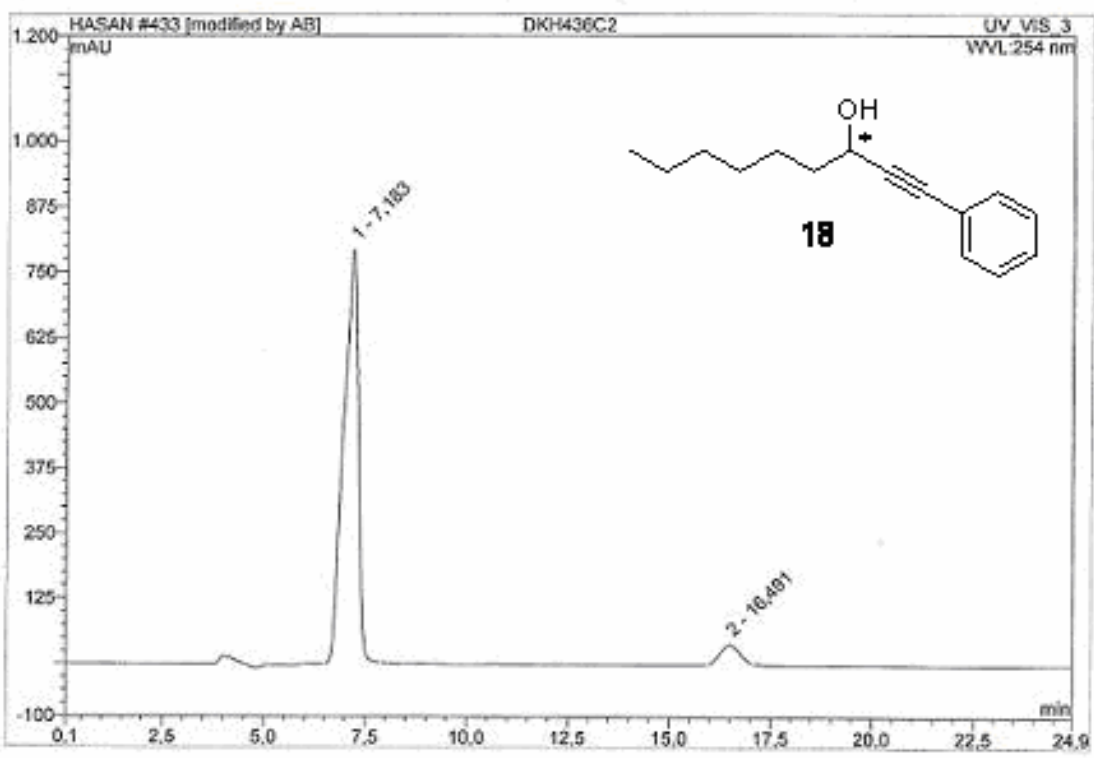

\begin{tabular}{|c|c|c|c|c|c|c|c|}
\hline No. & $\begin{array}{c}\text { Ret.Time } \\
\text { min }\end{array}$ & Peak Name & $\begin{array}{c}\text { Height } \\
\text { mAU }\end{array}$ & $\begin{array}{c}\text { Arca } \\
\text { mAU'min }\end{array}$ & $\begin{array}{c}\text { Rel_Area } \\
\%\end{array}$ & Amount & Type \\
\hline 1 & 7,18 & n.a. & 791,330 & 332,234 & 94,28 & n.a. & BMB $^{\circ}$ \\
\hline 2 & 16.49 & n.a. & 36,625 & 20,151 & 5.72 & n.a. & BMB: \\
\hline Total: & & & 827,955 & 352,386 & 100,00 & 0,000 & \\
\hline
\end{tabular}




\begin{tabular}{|llll|}
\hline 505 DKH470C2 & & \\
& & \\
\hline Sample Name: & DKH470C2 & Injection Volume: & 20,0 \\
Vial Number: & 520 & Channel: & UV_VIS_1 \\
Sample Type: & unknown & Wavelength: & $\mathbf{2 1 4}$ \\
Control Program: & PROGRAM01 & Bandwidth: & 2 \\
Quantif. Method: & METHOD01 & Dilution Factor: & $\mathbf{1 , 0 0 0 0}$ \\
Recording Time: & $\mathbf{1 5 . 3 . 2 0 0 7 ~ 1 9 : 5 8}$ & Sample Weight: & $\mathbf{1 , 0 0 0 0}$ \\
Run Time (min): & $\mathbf{1 4 , 8 8}$ & Sample Amount: & $\mathbf{1 , 0 0 0 0}$ \\
\hline
\end{tabular}

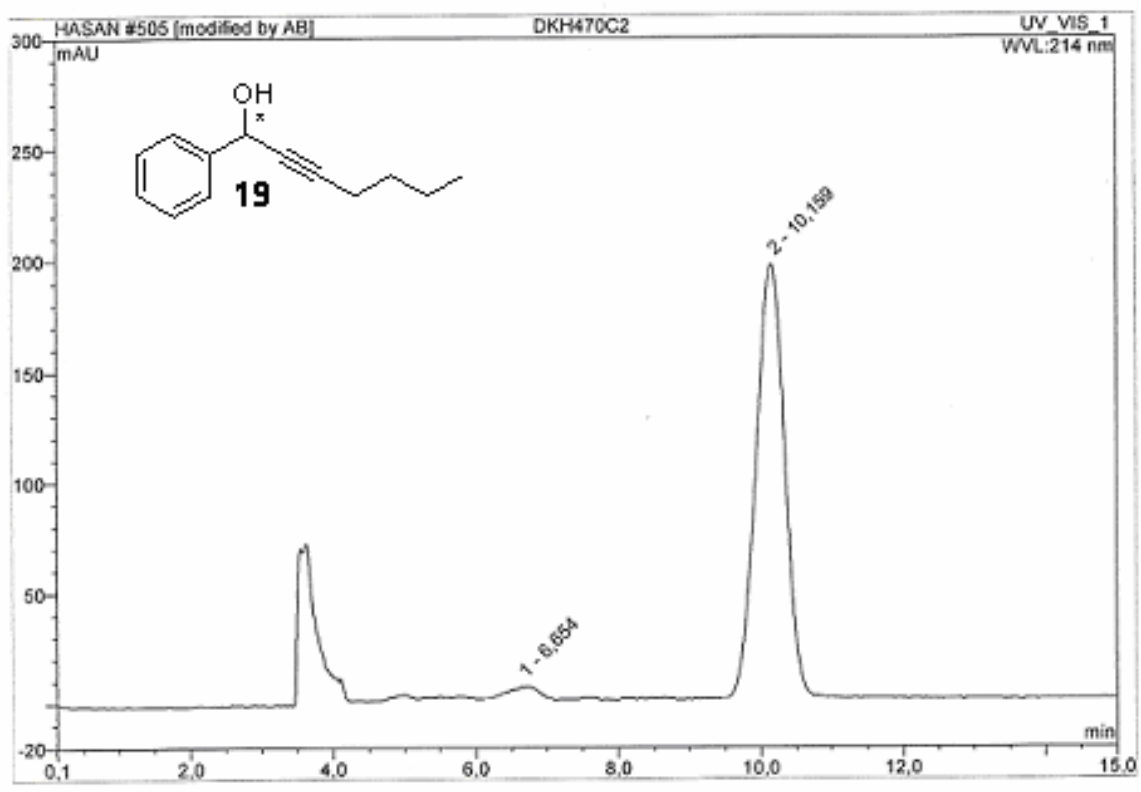

\begin{tabular}{|c|c|c|c|c|c|c|c|}
\hline No. & $\begin{array}{c}\text { Ret.Time } \\
\text { min }\end{array}$ & Peak Name & $\begin{array}{c}\text { Height } \\
\text { mAU }\end{array}$ & $\begin{array}{c}\text { Area } \\
\text { mAU*min }\end{array}$ & $\begin{array}{c}\text { Rel.Area } \\
\%\end{array}$ & Amount & Type \\
\hline 1 & 6,65 & n.a. & 5,341 & 2,513 & 2,69 & n.a. & BMB* $^{*}$ \\
\hline 2 & 10,16 & n.a. & 195,716 & 90,970 & 97,31 & n.a. & $\mathrm{BMB}^{*}$ \\
\hline Total: & & & 201,057 & 93,483 & 100,00 & 0,000 & \\
\hline
\end{tabular}




\section{DKH471C2}

\begin{tabular}{llll}
\hline Sample Name: & DKH471C2 & Injection Volume: & $\mathbf{2 0 , 0}$ \\
Vial Number: & $\mathbf{5 2 2}$ & Channel: & UV_VIS_1 \\
Sample Type: & unknown & Wavelength: & $\mathbf{2 1 4}$ \\
Control Program: & PROGRAM01 & Bandwidth: & $\mathbf{2}$ \\
Quantif. Method: & METHOD01 & Dilution Factor: & $\mathbf{1 , 0 0 0 0}$ \\
Recording Time: & $\mathbf{1 5 . 3 . 2 0 0 7 ~ 2 0 : 4 3}$ & Sample Weight: & $\mathbf{1 , 0 0 0 0}$ \\
Run Time (min): & $\mathbf{1 4 , 8 8}$ & Sample Amount: & $\mathbf{1 , 0 0 0 0}$
\end{tabular}

350 HASAN 5507 [modified by AB] $\quad$ DKH471C2 $\quad$ UV VIS 1

WVL214 210

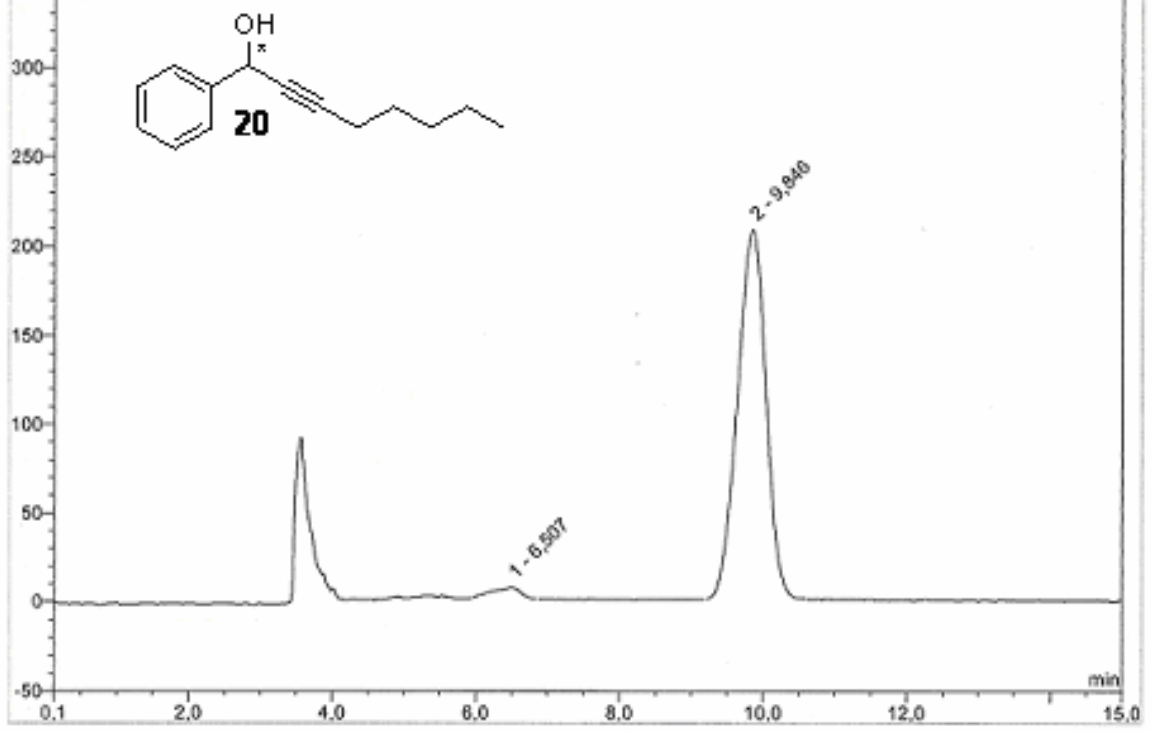

\begin{tabular}{|c|c|c|c|c|c|c|c|}
\hline No. & $\begin{array}{c}\text { Ret.Time } \\
\text { min }\end{array}$ & Peak Name & $\begin{array}{c}\text { Height } \\
\text { mAU }\end{array}$ & $\begin{array}{c}\text { Area } \\
\text { mAU*min }\end{array}$ & $\begin{array}{c}\text { Rel.Area } \\
\% \\
\end{array}$ & Amount & Type \\
\hline 1 & 6,51 & n.a. & 6,717 & 3,433 & 3,48 & n.a. & $\mathrm{MB}^{*}$ \\
\hline 2 & 9,85 & n.a. & 207,082 & 95,182 & 96,52 & n.a. & $\mathrm{BM}$ * \\
\hline Total: & & & 213,799 & 98,615 & 100,00 & 0,000 & \\
\hline
\end{tabular}

